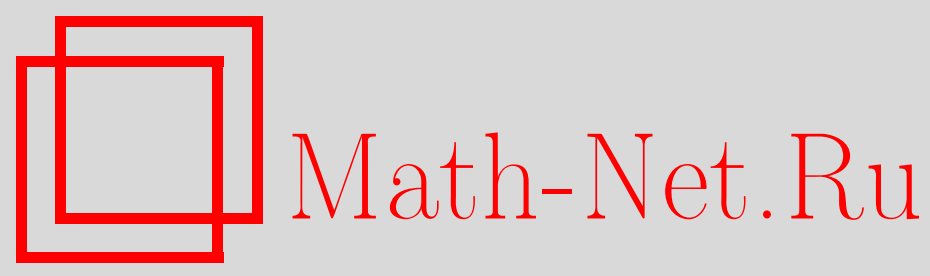

Ф. З. Рахмонов, Исследование особого ряда в проблеме Варинга-Гольдбаха со сдвинутыми простыми числами, Дискрет. матем., 2011, том 23, выпуск 4, 3-32

DOI: https://doi.org/10.4213/dm1158

Использование Общероссийского математического портала Math-Net.Ru подразумевает, что вы прочитали и согласны с пользовательским соглашением http://www.mathnet.ru/rus/agreement

Параметры загрузки:

IP : 3.91 .87 .62

26 апреля 2023 г., $17: 44: 14$ 


\title{
Исследование особого ряда в проблеме Варинга-Гольдбаха со сдвинутыми простыми числами
}

\author{
(C) 2011 г. Ф. З. Рахмонов
}

Доказано, что для особого ряда $\mathfrak{S}(N)$ в асимптотической формуле для числа представлений достаточно большого натурального числа $N, N \equiv 0(\bmod 4)$, в виде суммы пяти квадратов сдвинутых простых чисел $p_{i}+1$ справедливо соотношение

$$
\Im(N)=\left\{\begin{array}{lll}
c(N), & N \equiv 0 & (\bmod 4) ; \\
0, & N \neq 00 & (\bmod 4),
\end{array} \quad c(N)>2 \prod_{p \geqslant 7}\left(1-\frac{10}{\varphi^{2}(p)}\right)\right.
$$

\section{1. Введение}

В 1938 году Хуа Ло Ген [1] доказал асимптотическую формулу для числа представлений достаточно большого натурального числа $N$ в виде суммы пяти квадратов простых чисел и показал, что при $N \equiv 5(\bmod 24)$ особый ряд задачи больше абсолютной положительной постоянной, зависящей только от $N$, и равен нулю, если $N \not \equiv 5(\bmod 24)$. В работе исследуется особый ряд - аналог задачи Хуа Ло Гена, когда простое число $p$ заменяется на его сдвинутое $p+1$, а именно, найдено арифметическое условие, при выполнении которого особый ряд больше абсолютной положительной постоянной, зависящей только от $N$. Постановка этой задачи принадлежит В. Н. Чубарикову.

Мы будем использовать следующие обозначения.

Пусть $\operatorname{ord}_{p}(x)$ - наибольшая степень простого числа $p$, делящего целое число $x$, то есть $p^{\operatorname{ord}_{p}(x)} \| x$. Пусть, далее,

$$
\delta_{p}(n)=\left(\frac{n}{p}\right)
$$

- символ Лежандра,

$$
\varepsilon_{p}=\delta_{p}(-1)=\left(\frac{-1}{p}\right)=(-1)^{(p-1) / 2}
$$


$c_{q}(n)$ - сумма Рамануджана;

$$
\begin{aligned}
T_{2}(a, q) & =\sum_{\substack{n=1 \\
(n, q)=1}}^{q} e\left(\frac{a(n+1)^{2}}{q}\right), \\
\Phi(q, N) & =\frac{1}{\varphi^{5}(q)} \sum_{\substack{a=0 \\
(a, q)=1}}^{q} T_{2}^{5}(a, q) e\left(-\frac{a N}{q}\right), \\
\mathscr{F}(p, N) & =1+\sum_{\alpha=1}^{\infty} \Phi\left(p^{\alpha}, N\right), \\
\Im & (N)=\sum_{q=1}^{\infty} \Phi(q, N) .
\end{aligned}
$$

Здесь и далее $\varphi(p)$ есть функция Эйлера.

Теорема 1. Справедливо соотночение

$$
\mathfrak{S}(N)=\prod_{p} \mathscr{F}(p, N)=\left\{\begin{array}{lll}
c(N), & N \equiv 0 & (\bmod 4) ; \\
0, & N \neq 00 & (\bmod 4),
\end{array}\right.
$$

где с $(N)$ - абсолютная положительная постоянная, зависящая только от $N, u$

$$
c(N)>2 \prod_{p \geqslant 7}\left(1-\frac{10}{\varphi^{2}(p)}\right) .
$$

Основу доказательства теоремы 1 составляют теорема 2 о точном значении ряда $\mathscr{F}(2, N)$, ее следствие 1 об оценке снизу $\mathscr{F}(2, N)$ при $\operatorname{ord}_{2}(N) \geqslant 2$ и теорема 3 со своими следствиями $2,4,6$, в которых, соответственно, получены оценки снизу для $\mathscr{F}(p, N)$ при $p \geqslant 7, \mathscr{F}(3, N), \mathscr{F}(5, N)$. Доказательства теорем 2 и 3, в свою очередь, опираются на точные значения суммы $\Phi\left(a, p^{\alpha}\right)$, которые найдены, соответственно, в леммах 3,4 при $p=2$ и в леммах 9, 10, 11 при $p \geqslant 3$.

Теорема 2. Пусть $\operatorname{ord}_{2}(N)=\beta$, $\operatorname{ord}_{2}\left(N \cdot 2^{-\beta}-5\right)=\eta$, тогда справедлива формула

$$
\mathscr{F}(2, N)= \begin{cases}0, & \text { если } \beta \leqslant 1 ; \\ \frac{26}{7}+\frac{15}{28} 2^{-\frac{3}{2}(\beta-1)+3}, & \text { если } \beta \geqslant 2 u \beta \text { четно, } \eta \neq 2 ; \\ \frac{26}{7}-\frac{13}{28} 2^{-\frac{3}{2}(\beta-1)+3}, & \text { если } \beta \geqslant 2 u \beta \text { нечетно, } \eta=2 ; \\ \frac{26}{7}+\frac{15}{28} 2^{-\frac{3}{2}(\beta-1)+3}, & \text { если } \beta \geqslant 3 \text { и } \beta \text { нечетно. }\end{cases}
$$

Следствие 1. При $\operatorname{ord}_{2}(N) \geqslant 2$, справедливо неравенство

$$
\mathscr{F}(2, N) \geqslant \frac{26}{7}-\frac{13 \sqrt{2}}{14} \geqslant \frac{13}{14}(4-\sqrt{2})>\frac{65}{28} .
$$


Теорема 3. Пусть $p-$ нечетное простое число, $\operatorname{ord}_{p}(N)=\beta$, тогда справедлива формула

$$
\mathscr{F}(p, N)=1+\Phi(p, N)+\mathscr{F}_{1}(p, N),
$$

2de

$$
\begin{aligned}
\Phi(p, N)= & \frac{1}{\varphi^{5}(p)}\left(-c_{p}(N-5)-10 \varepsilon_{p} p c_{p}(N-3)-5 p^{2} c_{p}(N-1)\right. \\
& \left.+5 p \delta_{p}(N-4)+10 \varepsilon_{p} \delta_{p}(N-2) p^{2}+\delta_{p}(N) p^{3}\right), \\
\mathscr{F}_{1}(p, N)= & \begin{array}{ll}
0, & \text { если } \beta=0 ; \\
-\frac{p}{(p-1)^{5}} & \text { если } \beta \geqslant 1 \text { нечетно, } \\
\frac{p^{4}}{(p-1)^{5}\left(p^{3}-1\right)}\left(1-\frac{1}{\left.p^{\frac{3}{2}(\beta-1)}\right)}\right) & \text { если } \beta \geqslant 2 \text { четно. } \\
+\frac{1+\varepsilon_{p} \delta_{p}(N)}{(p-1)^{4}\left(p^{3}-1\right)}\left(1-\frac{(p+1)^{2}}{\left.p^{\frac{3}{2}(\beta-2)+4}\right)},\right. &
\end{array}
\end{aligned}
$$

Следствие 2. При $p \geqslant 7$ справедливо неравенство

$$
\mathscr{F}(p, N)>1-\frac{10}{\varphi^{2}(p)} .
$$

Следствие 3. Пусть $\operatorname{ord}_{3}(N)=\beta$, тогда справедлива формула

$$
\mathscr{F}(3, N)= \begin{cases}1-2^{-4}+\frac{15}{52}\left(1-\frac{1}{3^{\frac{3}{2}(\beta-1)}}\right), & \text { если } \beta \geqslant 1 \text { нечетно; } \\ 1+\frac{47}{208}-\frac{1}{26 \cdot 3^{\frac{3}{2}(\beta-2)}}+\frac{1-\delta_{3}(N)}{2^{5} \cdot 3^{\frac{3}{2}(\beta-2)}}, & \text { если } \beta \geqslant 2 \text { четно; } \\ 1+2^{-4}, & \text { если } N \equiv 1 \quad(\bmod 3) ; \\ 1+2^{-5}, & \text { если } N \equiv 2 \quad(\bmod 3) .\end{cases}
$$

Следствие 4. Справедливо неравенство

$$
\mathscr{F}(3, N) \geqslant 1-2^{-4} \text {. }
$$

Следствие 5. Пусть $\operatorname{ord}_{5}(N)=\beta$, тогда справедлива формула

$$
\mathscr{F}(5, N)= \begin{cases}1-\frac{59}{2^{10}}+\frac{5^{4}}{31 \cdot 2^{10}}\left(1-\frac{1}{5^{\frac{3}{2}(\beta-1)}}\right), & \text { если } \beta \geqslant 1 \text { нечетно; } \\
1-\frac{1049}{31 \cdot 2^{10}}-\frac{3^{2}}{31 \cdot 2^{8} \cdot 5^{\frac{3}{2}(\beta-2)}} & \\
+\frac{1+\delta_{5}(N)}{2^{10} \cdot 5^{\frac{3}{2}(\beta-2)},} & \text { если } \beta \geqslant 2 \text { четно; } \\
\begin{array}{ll}
1+99 \cdot 2^{-10}, \\
1+26 \cdot 2^{-10},
\end{array} & \text { если } N \equiv 1 \quad(\bmod 5) ; \\
1+76 \cdot 2^{-10}, & \text { если } N \equiv 2(\bmod 5) ; \\
1+51 \cdot 2^{-10}, & \text { если } N \equiv 3 \quad(\bmod 5) ; \\
\text { если } N \equiv 4 \quad(\bmod 5) .\end{cases}
$$


Следствие 6. Справедливо неравенство

$$
\mathscr{F}(5, N) \geqslant 1-\frac{59}{2^{10}} .
$$

\section{2. Вычисление $\mathscr{F}(2, N)$}

В этом параграфе мы докажем теорему 2 точном значении ряда $\mathscr{F}(2, N)$ и ее следствие 1 об оценке снизу ряда $\mathscr{F}(2, N)$ при $\beta=\operatorname{ord}_{2}(N) \geqslant 2$. Предварительно докажем вспомогательные леммы о точных значениях тригонометрических сумм $S\left(a, 2^{\alpha}\right), T_{2}\left(a, 2^{\alpha}\right)$, $\Phi\left(2^{\alpha}, N\right)$, которыми будем пользоваться при доказательстве теоремы 2.

Лемма 1. Пусть $(a, 2)=1, \alpha$ - натуральное число. Тогда справедлива формула

$$
S\left(a, 2^{\alpha}\right)=\sum_{n=1}^{2^{\alpha}} e\left(\frac{a n^{2}}{2^{\alpha}}\right)= \begin{cases}0, & \text { если } \alpha=1, \\ 2^{\alpha / 2}\left(1+i^{a}\right), & \text { если } \alpha-\text { четное } \\ 2^{\alpha / 2}(1+i)^{a}, & \text { если } \alpha-\text { нечетное } u \alpha \geqslant 3 ;\end{cases}
$$

Доказательство. Введем обозначение

$$
S(a, q, r)=\sum_{n=1}^{q} e\left(\frac{a n^{2}}{r}\right) .
$$

При $r=q$, сумма $S(a, q, r)$ превращается в сумму Гаусса по модулю $q$, то есть

$$
S(a, q, q)=S(a, q)
$$

Найдем точное значение $S(a, 2,4)$ и $S(a, 4,8)$. Справедливы равенства

$$
\begin{aligned}
S(a, 2,4) & =\sum_{n=1}^{2} e\left(\frac{a n^{2}}{4}\right)=1+e\left(\frac{a}{4}\right)=1+e^{\frac{\pi a}{2} i}=1+i^{a}, \\
S(a, 4,8) & =\sum_{n=1}^{4} e\left(\frac{a n^{2}}{8}\right)=2 e\left(\frac{a}{8}\right)+e\left(\frac{a}{2}\right)+1=2 e\left(\frac{a}{8}\right) \\
& =2 e^{\frac{\pi a}{4} i}=2\left(e^{\frac{\pi}{4} i}\right)^{a}=\sqrt{2}(1+i)^{a} .
\end{aligned}
$$

Преобразуя сумму $S\left(a, 2^{\alpha}\right)$ при $\alpha \geqslant 2$ подстановкой $n=n_{1} 2^{\alpha-1}+n_{2}$, где $n_{1}$ и $n_{2}$ независимо пробегают значения $n_{1}=0,1, n_{2}=1,2, \ldots, 2^{\alpha-1}$, и имея в виду, что

$$
\left(n_{1} 2^{\alpha-1}+n_{2}\right)^{2}=n_{1}^{2} 2^{2 \alpha-2}+n_{1} n_{2} 2^{\alpha}+n_{2}^{2} \equiv n_{2}^{2} \quad\left(\bmod 2^{\alpha}\right),
$$

получим, что

$$
\begin{aligned}
S\left(a, 2^{\alpha}\right) & =\sum_{n=1}^{2^{\alpha}} e\left(\frac{a n^{2}}{2^{\alpha}}\right)=\sum_{n_{1}=0}^{1} \sum_{n_{2}=1}^{2^{\alpha-1}} e\left(\frac{a\left(n_{1} 2^{\alpha-1}+n_{2}\right)^{2}}{2^{\alpha}}\right) \\
& =2 \sum_{n=1}^{2^{\alpha-1}} e\left(\frac{a n^{2}}{2^{\alpha}}\right)=2 S\left(a, 2^{\alpha-1}, 2^{\alpha}\right) .
\end{aligned}
$$


Пусть теперь $\alpha \geqslant 4$. Преобразуя сумму $S\left(a, 2^{\alpha-1}, 2^{\alpha}\right)$ подстановкой $n=n_{1} 2^{\alpha-2}+n_{2}$, где $n_{1}$ и $n_{2}$ независимо пробегают значения $n_{2}=1,2, \ldots, 2^{\alpha-2}, n_{1}=0,1$, и имея в виду, что

$$
\left(n_{1} 2^{\alpha-2}+n_{2}\right)^{2}=n_{1}^{2} 2^{2 \alpha-4}+n_{1} n_{2} 2^{\alpha-1}+n_{2}^{2} \equiv n_{1} n_{2} 2^{\alpha-1}+n_{2}^{2} \quad\left(\bmod 2^{\alpha}\right),
$$

находим, что

$$
\begin{aligned}
S\left(a, 2^{\alpha-1}, 2^{\alpha}\right) & =\sum_{n=1}^{2^{\alpha-1}} e\left(\frac{a n^{2}}{2^{\alpha}}\right)=\sum_{n_{1}=0}^{1} \sum_{n_{2}=1}^{2^{\alpha-2}} e\left(\frac{a\left(n_{1} 2^{\alpha-2}+n_{2}\right)^{2}}{2^{\alpha}}\right) \\
& =\sum_{n_{2}=1}^{2^{\alpha-2}} e\left(\frac{a n_{2}^{2}}{2^{\alpha}}\right) \sum_{n_{1}=0}^{1} e\left(\frac{a n_{1} n_{2}}{2}\right) \\
& =2 \sum_{n_{2}=0}^{2_{(\bmod 2)}^{\alpha-2}} e\left(\frac{a n_{2}^{2}}{2^{\alpha}}\right)=2 \sum_{n=1}^{2^{\alpha-3}} e\left(\frac{a n^{2}}{2^{\alpha-2}}\right)=2 S\left(a, 2^{\alpha-3}, 2^{\alpha-2}\right) .
\end{aligned}
$$

Следовательно, имеют место рекуррентные формулы

$$
\begin{aligned}
S\left(a, 2^{\alpha}\right) & =2 S\left(a, 2^{\alpha-1}, 2^{\alpha}\right), & & \alpha \geqslant 2, \\
S\left(a, 2^{\alpha-1}, 2^{\alpha}\right) & =2 S\left(a, 2^{\alpha-3}, 2^{\alpha-2}\right), & & \alpha \geqslant 4,
\end{aligned}
$$

Пусть $\alpha$ четно и $\alpha \geqslant 4$. Применим к сумме $S\left(a, 2^{\alpha}\right)$ формулу (3); далее, к полученной сумме $S\left(a, 2^{\alpha-1}, 2^{\alpha}\right)$ последовательно $(\alpha-2) / 2$ раз применим формулу (4) и получим, что

$$
\begin{aligned}
S\left(a, 2^{\alpha}\right) & =2 S\left(a, 2^{\alpha-1}, 2^{\alpha}\right)=2 \cdot 2 S\left(a, 2^{\alpha-3}, 2^{\alpha-2}\right)=2 \cdot 2^{2} S\left(a, 2^{\alpha-5}, 2^{\alpha-4}\right)=\cdots \\
& =2 \cdot 2^{j} S\left(a, 2^{\alpha-(2 j+1)}, 2^{\alpha-2 j}\right)=\cdots \\
& =2 \cdot 2^{\alpha / 2-1} S\left(a, 2^{\alpha-(2 \cdot(\alpha-2) / 2+1)}, 2^{\alpha-2 \cdot(\alpha-2) / 2}\right) \\
& =2 \cdot 2^{\alpha / 2-1} S\left(a, 2,2^{2}\right)=2^{\alpha / 2} S(a, 2,4) .
\end{aligned}
$$

Подставляя в правую часть этого равенства из (1) точное значение $S(a, 2,4)$, находим, что

$$
S\left(a, 2^{\alpha}\right)=2^{\alpha / 2}\left(1+i^{a}\right), \quad \alpha \geqslant 4, \quad \alpha \text { - четное. }
$$

Пусть теперь $\alpha$ нечетно и $\alpha \geqslant 5$. Поступая, как в предыдущем случае, применим к сумме $S\left(a, 2^{\alpha}\right)$ формулу (3), далее к полученной сумме $S\left(a, 2^{\alpha-1}, 2^{\alpha}\right)$ последовательно $(\alpha-3) / 2$ раз применяем формулу (4) и получаем, что

$$
\begin{aligned}
S\left(a, 2^{\alpha}\right) & =2 S\left(a, 2^{\alpha-1}, 2^{\alpha}\right)=2 \cdot 2 S\left(a, 2^{\alpha-3}, 2^{\alpha-2}\right)=2 \cdot 2^{2} S\left(a, 2^{\alpha-5}, 2^{\alpha-4}\right)=\cdots \\
& =2 \cdot 2^{j} S\left(a, 2^{\alpha-(2 j+1)}, 2^{\alpha-2 j}\right)=\cdots \\
& =2 \cdot 2^{\cdot(\alpha-3) / 2} S\left(a, 2^{\alpha-(2 \cdot(\alpha-3) / 2+1)}, 2^{\alpha-2 \cdot(\alpha-3) / 2}\right) \\
& =2 \cdot 2^{(\alpha-1) / 2-1} S\left(a, 2^{2}, 2^{3}\right)=2^{(\alpha-1) / 2} S(a, 4,8) .
\end{aligned}
$$

Подставляя в правую часть этого равенства из (2) точное значение $S(a, 4,8)$, находим, что

$$
S\left(a, 2^{\alpha}\right)=2^{\alpha / 2}(1+i)^{a}, \quad \alpha \geqslant 5, \quad \alpha \text { - нечетное. }
$$


При $\alpha=1,2,3$, пользуясь формулами (1) и (2), находим, что

$$
\begin{gathered}
S(a, 2)=e\left(\frac{a}{2}\right)+1=-1+1=0, \\
S\left(a, 2^{2}\right)=2 S\left(a, 2,2^{2}\right)=2\left(1+i^{a}\right), \\
S\left(a, 2^{3}\right)=2 S\left(a, 2^{2}, 2^{3}\right)=2^{3 / 2}\left(1+i^{a}\right) .
\end{gathered}
$$

Отсюда и из формул (5) и (6) следует утверждение леммы.

Лемма 2. Пусть $(a, 2)=1, \alpha-$ натуральное число. Тогда справедлива формула

$$
T_{2}\left(a, 2^{\alpha}\right)=\sum_{\substack{n=1 \\(n, 2)=1}}^{2^{\alpha}} e\left(\frac{a(n+1)^{2}}{2^{\alpha}}\right)= \begin{cases}1, & \text { если } \alpha=1 ; \\ 2, & \text { если } \alpha=2 ; \\ 0, & \text { если } \alpha=3 ; \\ 2^{\alpha / 2}\left(1+i^{a}\right), & \text { если } \alpha \geqslant 4 \text { четно; } \\ 2^{\alpha / 2}(1+i)^{a}, & \text { если } \alpha \geqslant 5 \text { нечетно. }\end{cases}
$$

Доказательство. В случае $\alpha=1,2,3$ вычислим сумму $T_{2}\left(a, 2^{\alpha}\right)$. Сумму $T_{2}\left(a, 2^{\alpha}\right)$ в случае $\alpha \geqslant 3$ выразим через сумму Гаусса. Для этого в $T_{2}\left(a, 2^{\alpha}\right)$ в переменной суммирования, которая пробегает все нечетные числа $n, 1 \leqslant n \leqslant 2^{\alpha}$, сделаем подстановку $n=2 m-1$, $1 \leqslant m \leqslant 2^{\alpha-1}$. Справедливы равенства

$$
\begin{aligned}
T_{2}\left(a, 2^{\alpha}\right) & =\sum_{\substack{n=1 \\
(n, 2)=1}}^{2^{\alpha}} e\left(\frac{a(n+1)^{2}}{2^{\alpha}}\right)=\sum_{m=1}^{2^{\alpha-1}} e\left(\frac{a((2 m-1)+1)^{2}}{2^{\alpha}}\right) \\
& =\sum_{m=1}^{2^{\alpha-1}} e\left(\frac{a m^{2}}{2^{\alpha-2}}\right)=\sum_{n=1}^{2^{\alpha-2}} e\left(\frac{a n^{2}}{2^{\alpha-2}}\right)+\sum_{n=1}^{2^{\alpha-2}} e\left(\frac{a\left(2^{\alpha-2}+n\right)^{2}}{2^{\alpha-2}}\right) \\
& =2 \sum_{n=1}^{2^{\alpha-2}} e\left(\frac{a n^{2}}{2^{\alpha-2}}\right)=2 S\left(a, 2^{\alpha-2}\right) .
\end{aligned}
$$

Если $\alpha \geqslant 4$ и $\alpha$ - четное, то, подставляя в эту формулу из леммы 1 точное значение суммы Гаусса $S\left(a, 2^{\alpha}\right)$ для четных $\alpha$, находим, что

$$
T_{2}\left(a, 2^{\alpha}\right)=2 S\left(a, 2^{\alpha-2}\right)=2 \cdot 2^{(\alpha-2) / 2}\left(1+i^{a}\right) .
$$

Если $\alpha \geqslant 5$ и $\alpha-$ нечетное, то подставляем из этой же леммы 1 точное значение суммы $S\left(a, 2^{\alpha}\right)$ для нечетных $\alpha$ и получаем, что

$$
T_{2}\left(a, 2^{\alpha}\right)=2 S\left(a, 2^{\alpha-2}\right)=2 \cdot 2^{(\alpha-2) / 2}(1+i)^{a}=2^{\frac{\alpha}{2}}(1+i)^{a}=2^{\frac{\alpha+1}{2}} e\left(\frac{a}{8}\right) .
$$

Лемма доказана.

Лемма 3. Пусть $\alpha \geqslant 4-$ четное число. Тогда справедлива формула

$$
\Phi\left(2^{\alpha}, N\right)= \begin{cases}0, & \text { если либо } \beta \leqslant 1, \text { либо } \alpha>\beta+2, \beta \geqslant 2 ; \\ -2^{-\frac{3 \alpha}{2}+4}, & \text { если } 4 \leqslant \alpha \leqslant \beta ; \\ 2^{-\frac{3 \beta-5}{2}}, & \text { если } \alpha=\beta+1 \text { и } \beta \text { нечетно; } \\ -(-1)^{\frac{N \cdot 2-\beta-5}{2}} 2^{-\frac{3 \beta}{2}+1}, & \text { если } \alpha=\beta+2 \text { и } \beta \text { четно; }\end{cases}
$$


Доказательство. Пользуясь утверждением леммы 2 о точном значении суммы $T_{2}\left(a, 2^{\alpha}\right)$ для четных $\alpha \geqslant 4$, находим, что

$$
\begin{aligned}
\Phi\left(2^{\alpha}, N\right) & =\frac{1}{\varphi^{5}\left(2^{\alpha}\right)} \sum_{\substack{a=1 \\
(a, 2)=1}}^{2^{\alpha}} T_{2}^{5}\left(a, 2^{\alpha}\right) e\left(-\frac{a N}{2^{\alpha}}\right)=\frac{1}{2^{5(\alpha-1)}} \sum_{\substack{a=1 \\
(a, 2)=1}}^{2^{\alpha}} 2^{\frac{5 \alpha}{2}}\left(1+i^{a}\right)^{5} e\left(-\frac{N a}{2^{\alpha}}\right) \\
& =2^{-\frac{5 \alpha}{2}+5} \sum_{b=0}^{2^{\alpha-2}-1}\left[\left(1+i^{4 b+1}\right)^{5} e\left(-\frac{N(4 b+1)}{2^{\alpha}}\right)+\left(1+i^{4 b+3}\right)^{5} e\left(-\frac{N(4 b+3)}{2^{\alpha}}\right)\right] \\
& =2^{-\frac{5 \alpha}{2}+5} \sum_{b=0}^{2^{\alpha-2}-1}\left[(1+i)^{5} e\left(-\frac{N}{2^{\alpha}}\right)+(1-i)^{5} e\left(-\frac{3 N}{2^{\alpha}}\right)\right] e\left(-\frac{N b}{2^{\alpha-2}}\right) \\
& =2^{-\frac{5 \alpha}{2}+5}\left[(1+i)^{5} e\left(-\frac{N}{2^{\alpha}}\right)+(1-i)^{5} e\left(-\frac{3 N}{2^{\alpha}}\right)\right]_{b=0}^{2^{\alpha-2}-1} e\left(-\frac{N b}{2^{\alpha-2}}\right) .
\end{aligned}
$$

Сумма по $b$ является полной линейной тригонометрической суммой по модулю $2^{\alpha-2}$, поэтому она и, следовательно, $\Phi\left(2^{\alpha}, N\right)$ равны нулю, если $N \neq \equiv 0\left(\bmod 2^{\alpha-2}\right)$, то есть $\Phi\left(2^{\alpha}, N\right)=0$, если $\alpha-2>\beta$. Таким образом, $\Phi\left(2^{\alpha}, N\right)=0$ при всех четных $\alpha>\beta+2$, в том числе, если $\beta \leqslant 1$, то $\Phi\left(2^{\alpha}, N\right)=0$ при всех четных $\alpha \geqslant 4$.

Если $N \equiv 0\left(\bmod 2^{\alpha-2}\right), \beta \geqslant 2$, то есть если $4 \leqslant \alpha \leqslant \beta+2$, то полная линейная тригонометрическая сумма по $b$ равна $2^{\alpha-2}$. Поэтому, имея в виду, что $N \cdot 2^{2-\alpha}-$ целое число, находим, что

$$
\begin{aligned}
\Phi\left(2^{\alpha}, N\right) & =2^{-\frac{3 \alpha}{2}+3}\left[(1+i)^{5} e\left(-\frac{N \cdot 2^{2-\alpha}}{2^{2}}\right)+(1-i)^{5} e\left(-\frac{3 N \cdot 2^{2-\alpha}}{2^{2}}\right)\right] \\
& =2^{-\frac{3 \alpha-11}{2}}\left[e\left(\frac{5}{8}\right) e\left(-\frac{N \cdot 2^{2-\alpha}}{2^{2}}\right)+e\left(-\frac{5}{8}\right) e\left(\frac{N \cdot 2^{2-\alpha}}{2^{2}}\right)\right] \\
& =2^{-\frac{3 \alpha-11}{2}}\left[e\left(\frac{5-N \cdot 2^{3-\alpha}}{2^{3}}\right)+e\left(\frac{N \cdot 2^{3-\alpha}-5}{2^{3}}\right)\right] \\
& =2^{-\frac{3 \alpha-9}{2}} \cos \left(\frac{\pi\left(N \cdot 2^{3-\alpha}-5\right)}{4}\right)=2^{-\frac{3 \alpha-9}{2}} \cos \left(\frac{\pi N \cdot 2^{2-\alpha}}{2}-\frac{5 \pi}{4}\right) \\
& =2^{-\frac{3 \alpha-9}{2}}\left(\cos \frac{\pi N \cdot 2^{2-\alpha}}{2} \cos \frac{5 \pi}{4}+\sin \frac{\pi N \cdot 2^{2-\alpha}}{2} \sin \frac{5 \pi}{4}\right) \\
& =-2^{-\frac{3 \alpha}{2}+4}\left(\cos \frac{\pi N \cdot 2^{2-\alpha}}{2}+\sin \frac{\pi N \cdot 2^{2-\alpha}}{2}\right) .
\end{aligned}
$$

Теперь, в зависимости от четности $\beta, \beta \geqslant 2$, найдем точное значение $\Phi\left(2^{\alpha}, N\right)$ для четных $\alpha$. Пусть $\beta$ - четное, тогда для $4 \leqslant \alpha \leqslant \beta+2$

$$
\begin{aligned}
\Phi\left(2^{\alpha}, N\right) & =-2^{-\frac{3 \alpha}{2}+4} \cos \frac{\pi N \cdot 2^{2-\alpha}}{2} \\
& =-2^{-\frac{3 \alpha}{2}+4} \cos \left(\pi N \cdot 2^{-\beta} \cdot 2^{1+(\beta-\alpha)}\right) \\
& =-2^{-\frac{3 \alpha}{2}+4}, \quad 4 \leqslant \alpha \leqslant \beta,
\end{aligned}
$$




$$
\begin{aligned}
\Phi\left(2^{\beta+2}, N\right) & =-2^{-\frac{3 \beta}{2}+1} \sin \frac{\pi N \cdot 2^{-\beta}}{2} \\
& =-2^{-\frac{3 \beta}{2}+1}(-1)^{\frac{N \cdot 2^{-\beta}-1}{2}} \\
& =-(-1)^{\left(N \cdot 2^{-\beta}-5\right) 2^{-1}} 2^{-\frac{3 \beta}{2}+1} .
\end{aligned}
$$

Пусть теперь $\beta-$ нечетное и $\beta \geqslant 3$, тогда для $4 \leqslant \alpha \leqslant \beta+1$ находим, что

$$
\begin{aligned}
\Phi\left(2^{\alpha}, N\right) & =-2^{-\frac{3 \alpha}{2}+4} \cos \frac{\pi N \cdot 2^{2-\alpha}}{2} \\
& =-2^{-\frac{3 \alpha}{2}+4} \cos \left(\pi N \cdot 2^{-\beta+1+(\beta-\alpha)}\right) \\
& =-2^{-\frac{3 \alpha}{2}+4}, \quad 4 \leqslant \alpha \leqslant \beta-1, \\
\Phi\left(2^{\beta+1}, N\right) & =-2^{-\frac{3}{2}(\beta+1)+4} \cos \frac{\pi N \cdot 2^{2-(\beta+1)}}{2} \\
& =-2^{-\frac{3 \beta}{2}+\frac{5}{2}} \cos \left(\pi N \cdot 2^{-\beta}\right) \\
& =2^{-\frac{3 \beta-5}{2}} .
\end{aligned}
$$

Лемма 4. Пусть $\alpha \geqslant 5$ - нечетное число. Тогда справедлива формула

$$
\Phi\left(2^{\alpha}, N\right)=2^{-(3 \beta-4) / 2}(-1)^{\left(N \cdot 2^{-\beta}-5\right) 2^{-2}},
$$

если $\alpha=\beta+3, \operatorname{ord}_{2}\left(N \cdot 2^{-\beta}-5\right) \geqslant 2$ и $\beta \geqslant 2-$ четное число, $и \Phi\left(2^{\alpha}, N\right)=0$ в противном случае.

Доказательство. Пользуясь леммой 2 о точном значении суммы $T_{2}\left(a, 2^{\alpha}\right)$ для нечетных $\alpha \geqslant 5$, находим, что

$$
\begin{aligned}
\Phi\left(2^{\alpha}, N\right) & =\frac{1}{\varphi^{5}\left(2^{\alpha}\right)} \sum_{\substack{a=1 \\
(a, 2)=1}}^{2^{\alpha}} T_{2}^{5}\left(a, 2^{\alpha}\right) e\left(-\frac{a N}{2^{\alpha}}\right) \\
& =\frac{1}{2^{5(\alpha-1)}} \sum_{\substack{a=1 \\
(a, 2)=1}}^{2^{\alpha}} 2^{\frac{5(\alpha+1)}{2}} e\left(\frac{5 a}{8}\right) e\left(-\frac{N a}{2^{\alpha}}\right) .
\end{aligned}
$$

Преобразуя последнюю сумму подстановкой $a=a_{1}+2^{3} a_{2}$, где $a_{1}$ и $a_{2}$ независимо пробегают значения $a_{1}=1,3,5,7, a_{2}=1,2, \ldots, 2^{\alpha-3}$, находим, что

$$
\begin{aligned}
\Phi\left(2^{\alpha}, N\right) & =2^{-\frac{5(\alpha-3)}{2}} \sum_{\substack{a_{1}=1 \\
\left(a_{1}, 2\right)=1}}^{2^{3}} \sum_{a_{2}=1}^{2^{\alpha-3}} e\left(\frac{\left(5\left(a_{1}+2^{3} a_{2}\right)\right.}{2^{3}}\right) e\left(-\frac{\left(N\left(a_{1}+2^{3} a_{2}\right)\right.}{2^{\alpha}}\right) \\
& =2^{-\frac{5(\alpha-3)}{2}} \sum_{\substack{a_{1}=1 \\
\left(a_{1}, 2\right)=1}}^{2^{3}} \sum_{a_{2}=1}^{2^{\alpha-3}} e\left(\frac{5 a_{1}}{2^{3}}\right) e\left(-\frac{N\left(a_{1}+2^{3} a_{2}\right)}{2^{\alpha}}\right) \\
& =2^{-\frac{5(\alpha-3)}{2}} \sum_{b=1}^{2^{2}} \sum_{a_{2}=1}^{2^{\alpha-3}} e\left(\frac{5(2 b-1)}{2^{3}}\right) e\left(-\frac{N\left(2 b-1+2^{3} a_{2}\right)}{2^{\alpha}}\right)
\end{aligned}
$$




$$
\begin{aligned}
& =2^{-\frac{5(\alpha-3)}{2}} \sum_{b=1}^{2^{2}} e\left(\frac{5(2 b-1)}{2^{3}}\right) e\left(\frac{-N(2 b-1)}{2^{\alpha}}\right) \sum_{a_{2}=1}^{2^{\alpha-3}} e\left(-\frac{N a_{2}}{2^{\alpha-3}}\right) \\
& =2^{-\frac{5(\alpha-3)}{2}} \sum_{b=1}^{2^{2}} e\left(\frac{\left(5-N \cdot 2^{3-\alpha}\right)(2 b-1)}{2^{3}}\right) \sum_{a_{2}=1}^{2^{\alpha-3}} e\left(-\frac{N a_{2}}{2^{\alpha-3}}\right) .
\end{aligned}
$$

Сумма по $a_{2}$ является полной линейной тригонометрической суммой по модулю $2^{\alpha-3}$, поэтому она и, следовательно, $\Phi\left(2^{\alpha}, N\right)$, равны нулю, если $N \not \equiv 0\left(\bmod 2^{\alpha-3}\right)$, то есть $\Phi\left(2^{\alpha}, N\right)=0$, если $\alpha-3>\beta$. Таким образом, $\Phi\left(2^{\alpha}, N\right)=0$ при всех нечетных $\alpha>\beta+3$, в том числе если $\beta \leqslant 1$, то $\Phi\left(2^{\alpha}, N\right)=0$ при всех нечетных $\alpha \geqslant 5$.

Если $N \equiv 0\left(\bmod 2^{\alpha-3}\right)$ и $\beta \geqslant 2$, то есть если $5 \leqslant \alpha \leqslant \beta+3$, то полная линейная тригонометрическая сумма по $a_{2}$ равна $2^{\alpha-3}$. Поэтому, имея в виду, что $N \cdot 2^{3-\alpha}-$ целое число, находим, что

$$
\begin{aligned}
\Phi\left(2^{\alpha}, N\right) & =2^{-\frac{3(\alpha-3)}{2}} \sum_{b=1}^{2^{2}} e\left(\frac{\left(5-N \cdot 2^{3-\alpha}\right)(2 b-1)}{2^{3}}\right) \\
& =2^{-\frac{3(\alpha-3)}{2}} e\left(\frac{N \cdot 2^{3-\alpha}-5}{2^{3}}\right) \sum_{b=1}^{2^{2}} e\left(\frac{\left(5-N \cdot 2^{3-\alpha}\right) b}{2^{2}}\right) .
\end{aligned}
$$

Отсюда получаем, что $\Phi\left(2^{\alpha}, N\right)=0$, если $\operatorname{ord}_{2}\left(N \cdot 2^{3-\alpha}-5\right)<2$. Если же выполняется соотношение $\operatorname{ord}_{2}\left(N \cdot 2^{3-\alpha}-5\right) \geqslant 2$, то, следовательно, $N \cdot 2^{3-\alpha}-5$ - четное число. Отсюда в свою очередь следует, что $N \cdot 2^{3-\alpha}$ нечетно. Число $N \cdot 2^{3-\alpha}$ с учетом условия $\alpha-3 \leqslant \beta$ будет нечетным только в том случае, если $2^{\alpha-3} \| N$, то есть если $\alpha-3=\beta$. Последнее равенство возможно только при четном $\beta$. Следовательно, $\Phi\left(2^{\alpha}, N\right)=0$ для

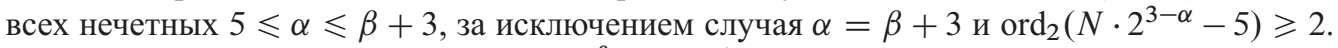
В этом случае, имея в виду, что $\left(N \cdot 2^{-\beta}-5\right) 2^{-2}-$ целое число, получаем, что

$$
\begin{aligned}
\Phi\left(2^{\beta+3}, N\right) & =2^{-\frac{3 \beta}{2}+2} e\left(\frac{N \cdot 2^{-\beta}-5}{2^{3}}\right)=2^{-\frac{3 \beta}{2}+2} e\left(\frac{\left(N \cdot 2^{-\beta}-5\right) 2^{-2}}{2}\right) \\
& =2^{-\frac{3 \beta-4}{2}}(-1)^{\left(N \cdot 2^{-\beta}-5\right) 2^{-2}} .
\end{aligned}
$$

Доказательство теоремы 2. Вычислим $\Phi\left(2^{\alpha}, N\right)$ для $\alpha=1,2,3$. Подставляя найденные в лемме 2 значения $T_{2}\left(a, 2^{\alpha}\right)$ соответственно в выражения для $\Phi\left(2^{\alpha}, N\right)$, находим, что

$$
\begin{aligned}
\Phi(2, N) & =\frac{1}{\varphi^{5}(2)} \sum_{\substack{a=1 \\
(a, 2)=1}}^{2} T_{2}^{5}(a, 2) e\left(-\frac{a N}{2}\right)=e\left(-\frac{N}{2}\right)=e\left(-\frac{2 N}{4}\right), \\
\Phi\left(2^{2}, N\right) & =\frac{1}{\varphi^{5}(4)} \sum_{\substack{a=1 \\
(a, 2)=1}}^{4} T_{2}^{5}(a, 4) e\left(-\frac{a N}{4}\right)=\sum_{\substack{a=1 \\
(a, 2)=1}}^{4} e\left(\frac{-N a}{2^{2}}\right) \\
& =e\left(\frac{-N}{4}\right)+e\left(\frac{-3 N}{4}\right), \\
\Phi\left(2^{3}, N\right) & =0 .
\end{aligned}
$$

Теперь, с помощью этих формул, найденных значений $\Phi\left(2^{\alpha}, N\right)$ для четных $\alpha \geqslant 4$ в лемме 3 и для нечетных $\alpha \geqslant 5$ в лемме 4 , вычислим значение $\mathscr{F}(2, N)$. Рассмотрим следующие три возможных случая. 
(i) $\beta \leqslant 1$. В этом случае из лемм 3 и 4 следует, что $\Phi\left(2^{\alpha}, N\right)=0$ при $\alpha \geqslant 4$, а значение $\Phi\left(2^{\alpha}, N\right)$ для $\alpha=1,2,3$ определяется с помощью формул (12), (13) и (14). Поэтому

$$
\begin{aligned}
\mathscr{F}(2, N) & =1+\sum_{\alpha=1}^{\infty} \Phi\left(2^{\alpha}, N\right)=1+\Phi(2, N)+\Phi\left(2^{2}, N\right) \\
& =1+e\left(\frac{-N}{4}\right)+e\left(\frac{-2 N}{4}\right)+e\left(\frac{-3 N}{4}\right)=0 .
\end{aligned}
$$

(ii) $\beta \geqslant 2$ четно. $\quad$ В этом случае из лемм 3 и 4 следует, что $\Phi\left(2^{\alpha}, N\right)=0$ при $\alpha>\beta+3$ и при всех нечетных $\alpha, 5 \leqslant \alpha \leqslant \beta+1$, а $\Phi\left(2^{\alpha}, N\right)$ определяется при $\alpha=1,2,3$ так же, как и в случае (i) с помощью формул (12, (13) и (14), при четных $\alpha, 4 \leqslant \alpha \leqslant \beta+2,-$ леммой 3 , при $\alpha=\beta+3-$ леммой 4 . Поэтому, вводя для $\Phi\left(2^{\beta+3}, N\right)$ характеристическую функцию

$$
\delta(\beta, N)= \begin{cases}1, & \text { если } \eta \geqslant 2 ; \\ 0, & \text { если } \eta=1,\end{cases}
$$

где

$$
\eta=\operatorname{ord}_{2}\left(N \cdot 2^{-\beta}-5\right) \geqslant 1
$$

(разность двух нечетных чисел делится на 2), и пользуясь соотношениями

$$
\begin{aligned}
& (-1)^{\left(N \cdot 2^{-\beta}-5\right) 2^{-1}}=(-1)^{2^{\eta-1}}, \\
& (-1)^{\left(N \cdot 2^{-\beta}-5\right) 2^{-2}}=(-1)^{2^{\eta-2}},
\end{aligned}
$$

находим, что

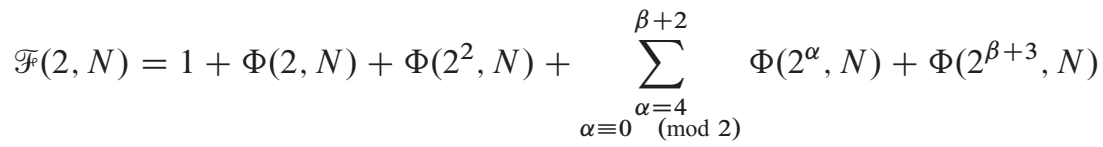

$$
\begin{aligned}
& =1+e\left(\frac{-N}{4}\right)+e\left(\frac{-2 N}{4}\right)+e\left(\frac{-3 N}{4}\right)-\sum_{\alpha \equiv 0}^{\beta=4} \underset{\substack{\bmod 2)}}{\beta} 2^{-\frac{3 \alpha}{2}+4} \\
& -(-1)^{\left(N \cdot 2^{-\beta}-5\right) 2^{-1}} 2^{-\frac{3 \beta}{2}+1}+\delta(\beta, N)(-1)^{\left(N \cdot 2^{-\beta}-5\right) 2^{-2}} 2^{-\frac{3 \beta-4}{2}} \\
& =4-\sum_{\alpha_{1}=2}^{\frac{1}{2} \beta} 2^{-3 \alpha_{1}+4}-(-1)^{2^{\eta-1}} 2^{-\frac{3}{2} \beta+1}+\delta(\beta, N)(-1)^{2^{\eta-2}} 2^{-\frac{3}{2} \beta+2} \\
& =4-2^{-2} \sum_{\alpha_{1}=0}^{\frac{1}{2} \beta-2} 2^{-3 \alpha_{1}}-(-1)^{2^{\eta-1}} 2^{-\frac{3}{2} \beta+1}+\delta(\beta, N)(-1)^{2^{\eta-2}} 2^{-\frac{3}{2} \beta+2} \\
& =4-\frac{1-\left(2^{-3}\right)^{\frac{1}{2} \beta-1}}{2^{2}\left(1-2^{-3}\right)}-(-1)^{2^{\eta-1}} 2^{-\frac{1}{2} \beta+1}+\delta(\beta, N)(-1)^{2^{\eta-2}} 2^{-\frac{3}{2} \beta+2} \\
& =4-\frac{2}{7}+\frac{2^{-\frac{3}{2} \beta+4}}{7}-(-1)^{2^{\eta-1}} 2^{-\frac{3}{2} \beta+1}+\delta(\beta, N)(-1)^{2^{\eta-2}} 2^{-\frac{3}{2} \beta+2} \\
& =\frac{26}{7}+\left(\frac{2}{7}-\frac{(-1)^{2^{\eta-1}}}{4}+\frac{\delta(\beta, N)(-1)^{2^{\eta-2}}}{2}\right) 2^{-\frac{3}{2}(\beta-1)+3} .
\end{aligned}
$$


Если $\eta=1$, то, имея в виду, что $\delta(\beta, N)=0$, находим, что

$$
\begin{aligned}
\mathscr{F}(2, N) & =\frac{26}{7}+\left(\frac{2}{7}+\frac{1}{4}\right) 2^{-\frac{3}{2}(\beta-1)+3} \\
& =\frac{26}{7}+\frac{15}{28} 2^{-\frac{3}{2}(\beta-1)+3} .
\end{aligned}
$$

Если $\eta=2$, то

$$
\begin{aligned}
\mathscr{F}(2, N) & =\frac{26}{7}+\left(\frac{2}{7}-\frac{1}{4}-\frac{1}{2}\right) 2^{-\frac{3}{2}(\beta-1)+3} \\
& =\frac{26}{7}-\frac{13}{28} 2^{-\frac{3}{2}(\beta-1)+3} .
\end{aligned}
$$

Если же $\eta \geqslant 3$, то

$$
\begin{aligned}
\mathscr{F}(2, N) & =\frac{26}{7}+\left(\frac{2}{7}-\frac{1}{4}+\frac{1}{2}\right) 2^{-\frac{3}{2}(\beta-1)+3} \\
& =\frac{26}{7}+\frac{15}{28} 2^{-\frac{3}{2}(\beta-1)+3} .
\end{aligned}
$$

(iii) $\beta \geqslant 3$ нечетно. В этом случае из лемм 3 и 4 следует, что $\Phi\left(2^{\alpha}, N\right)=0$ при $\alpha>\beta+2$ и при всех нечетных $\alpha, 5 \leqslant \alpha \leqslant \beta+2$, а $\Phi\left(2^{\alpha}, N\right)$ определяется при $\alpha=1,2,3$ так же, как в предыдущих случаях, с помощью формул (12), (13) и (14) и при четных $\alpha$, $4 \leqslant \alpha \leqslant \beta+1$ леммой 3. Следовательно,

$$
\begin{aligned}
\mathscr{F}(2, N) & =1+\Phi(2, N)+\Phi\left(2^{2}, N\right)+\sum_{\alpha \equiv 0) \underset{(\bmod 2)}{\beta+1} \Phi\left(2^{\alpha}, N\right)} \\
& =1+e\left(\frac{-N}{4}\right)+e\left(\frac{-2 N}{4}\right)+e\left(\frac{-3 N}{4}\right)-\sum_{\alpha \equiv 0}^{\beta-1} 2_{(\bmod 2)}^{-\frac{3 \alpha}{2}+4}+2^{-\frac{3 \beta-5}{2}} \\
& =4-\sum_{\alpha_{1}=2}^{\frac{1}{2}(\beta-1)-2} 2^{-3 \alpha_{1}+4}+2^{-\frac{3}{2}(\beta-1)+1}=4-2^{-2}\left(2^{-3}\right)^{\alpha_{1}}+2^{-\frac{3}{2}(\beta-1)+1} \\
& =4-\frac{1-\left(2^{-3}\right)^{\frac{1}{2}(\beta-1)-1}}{2^{2}\left(1-2^{-3}\right)}+2^{-\frac{3}{2}(\beta-1)+1}=4-\frac{2-2^{-\frac{3}{2}(\beta-1)+4}}{7}+2^{-\frac{3}{2}(\beta-1)+1} \\
& =4-\frac{2}{7}+\frac{2^{-\frac{3}{2}(\beta-1)+4}}{7}+2^{-\frac{3}{2}(\beta-1)+1}=\frac{26}{7}+\left(\frac{2}{7}+\frac{1}{4}\right) 2^{-\frac{3}{2}(\beta-1)+3} \\
& =\frac{26}{7}+\frac{15}{28} 2^{-\frac{3}{2}(\beta-1)+3} .
\end{aligned}
$$

Теорема доказана.

Доказательство следствия 1. Из теоремы 2 при $\operatorname{ord}_{2}(N)=\beta \geqslant 2$, четном $\beta$ и $\eta=2$, полагая $\beta=2$, получаем, что

$$
\mathscr{F}(2, N) \geqslant \frac{26}{7}-\frac{13}{28} 2^{-\frac{3}{2}(\beta-1)+3} \geqslant \frac{26}{7}-\frac{13}{28} 2^{\frac{3}{2}} \geqslant \frac{26}{7}-\frac{13 \sqrt{2}}{14} \geqslant \frac{13}{14}(4-\sqrt{2})>\frac{65}{28} .
$$




\section{3. Вычисление $\mathscr{F}(p, N), p>2$}

В этом параграфе для нечетных простых $p$ доказывается теорема 3 о точном значении ряда $\mathscr{F}(p, N)$ и ее следствия $5,7,9$, в которых, соответственно, получены оценки снизу для $\mathscr{F}(p, N)$ при $p \geqslant 7, \mathscr{F}(3, N), \mathscr{F}(5, N)$. Предварительно мы приведем вспомогательные леммы о точных значениях тригонометрических сумм $V\left(a, p^{\alpha}\right), T_{2}\left(a, p^{\alpha}\right), \Phi\left(p^{\alpha}, N\right)$.

Лемма 5 ([2]). Пусть р-нечетное простое число, тогда для суммы Гаусса справедлива следующая формула:

$$
\begin{aligned}
S\left(a, p^{\alpha}\right) & =\sum_{n=1}^{p^{\alpha}} e\left(\frac{a n^{2}}{p^{\alpha}}\right)=\delta_{p}^{\alpha}(a) i^{\left(\frac{p^{\alpha}-1}{2}\right)^{2}} p^{\frac{\alpha}{2}} \\
& = \begin{cases}\delta_{p}(a) i^{\left(\frac{p-1}{2}\right)^{2}} p^{\frac{\alpha}{2}}, & \text { если } \alpha \text { нечетно, } \\
p^{\frac{\alpha}{2}}, & \text { если } \alpha \text { четно. }\end{cases}
\end{aligned}
$$

Докажем лемму о точном значении суммы Рамануджана

$$
\begin{aligned}
& c_{q}(n)=\sum_{\substack{a=1 \\
(a, q)=1}}^{q} e\left(\frac{n a}{q}\right)=\sum_{d \backslash(q, n)} \mu\left(\frac{q}{d}\right) d, \\
& c_{p}(n)= \begin{cases}-1, & \text { если }(n, p)=1, \\
p-1, & \text { если }(n, p)=p,\end{cases}
\end{aligned}
$$

когда ее модуль равен степени простого числа.

Лемма 6. Пусть $p$ - нечетное простое число, $\alpha \geqslant 2, p^{\operatorname{ord}_{p}(N)} \| N, \operatorname{ord}_{p}(N) \leqslant \alpha-1$. Тогда для суммы Рамануджана $c_{p^{\alpha}}(N)$ справедлива формула

$$
c_{p^{\alpha}}(N)=\sum_{\substack{a=1 \\(a, p)=1}}^{p^{\alpha}} e\left(\frac{N a}{p^{\alpha}}\right)=p^{\operatorname{ord}_{p}(N)} \mu\left(p^{\alpha-\operatorname{ord}_{p}(N)}\right) .
$$

Доказательство. Преобразуя сумму $c_{p^{\alpha}}(N)$ подстановкой $a_{1}+a_{2} p^{\alpha-\operatorname{ord}_{p}(N)}$, где $a_{1}$ и $a_{2}$ независимо пробегают значения $a_{1}=1,2, \ldots, p^{\alpha-\operatorname{ord}_{p}(N)}, a_{2}=0,1, \ldots, p^{\operatorname{ord}_{p}(N)}-1$ и пользуясь представлением $N_{1}=N p^{-\operatorname{ord}_{p}(N)}$, получим, что

$$
\begin{aligned}
c_{p^{\alpha}}(N) & =\sum_{\substack{a=1 \\
(a, p)=1}}^{p^{\alpha}} e\left(\frac{N_{1} a}{p^{\alpha-\operatorname{ord} p(N)}}\right)=\sum_{\substack{a_{1}=1 \\
\left(a_{1}, p\right)=1}}^{p^{\alpha-\operatorname{ord} p(N)}} \sum_{a_{2}=0}^{p^{\operatorname{ord} p(N)}-1} e\left(\frac{N_{1}\left(a_{1}+a_{2} p^{\alpha-\operatorname{ord}_{p}(N)}\right.}{p^{\alpha-\operatorname{ord}_{p}(N)}}\right) \\
& =p^{\operatorname{ord}_{p}(N)} \sum_{\substack{a_{1}=1 \\
\left(a_{1}, p\right)=1}}^{p^{\alpha-\operatorname{ord} p(N)}} e\left(\frac{N_{1} a_{1}}{p^{\alpha-\operatorname{ord}_{p}(N)}}\right) \\
& =p^{\operatorname{ord}_{p}(N)} \mu\left(p^{\alpha-\operatorname{ord}_{p}(N)}\right) .
\end{aligned}
$$


Лемма 7. Пусть $p$ - нечетное простое число, $\alpha \geqslant 2$, $p^{\operatorname{ord}_{p}(N)} \| N$. Тогда справедлива формула

$$
\begin{aligned}
V\left(p^{\alpha}, N\right) & =\sum_{\substack{a=1 \\
(a, p)=1}}^{p^{\alpha}} \delta_{p}(a) e\left(-\frac{a N}{p^{\alpha}}\right) \\
& = \begin{cases}\delta_{p}\left(N p^{-\operatorname{ord}_{p}(N)}\right) i^{\left(\frac{p-1}{2}\right)^{2}} p^{\operatorname{ord}_{p}(N)+\frac{1}{2}}, & \text { если } \alpha=\operatorname{ord}_{p}(N)+1, \\
0, & \text { если } \alpha \neq \operatorname{ord}_{p}(N)+1 .\end{cases}
\end{aligned}
$$

Доказательство. Если $\alpha \leqslant \operatorname{ord}_{p}(N)$, то

$$
V\left(p^{\alpha}, N\right)=\sum_{\substack{a=1 \\(a, p)=1}}^{p^{\alpha}} \delta_{p}(a)=p^{\alpha-1} \sum_{a=1}^{p-1} \delta_{p}(a)=0 .
$$

Пусть $\alpha>\operatorname{ord}_{p}(N) \geqslant 1$ и $N=p^{\operatorname{ord}_{p}(N)} N_{1}$, тогда

$$
\begin{aligned}
& V\left(p^{\alpha}, N\right)=\sum_{\substack{a=1 \\
(a, p)=1}}^{p^{\alpha}} \delta_{p}(a) e\left(\frac{-a N_{1}}{p^{\alpha-\operatorname{ord} p(N)}}\right) \\
& =\sum_{\substack{a_{1}=1 \\
\left(a_{1}, p\right)=1}}^{p^{\alpha-\operatorname{ord} p(N)}} \sum_{a_{2}=0}^{p^{\operatorname{ord} p(N)}-1} \delta_{p}\left(a_{1}+a_{2} p^{\alpha-\operatorname{ord} p(N)}\right) e\left(-\frac{\left(a_{1}+a_{2} p^{\alpha-\operatorname{ord}_{p}(N)}\right) N_{1}}{p^{\alpha-\operatorname{ord}_{p}(N)}}\right) \\
& =\sum_{\substack{a_{1}=1 \\
\left(a_{1}, p\right)=1}}^{p^{\alpha-\operatorname{ord} p(N)}} \delta_{p}\left(a_{1}\right) e\left(-\frac{a_{1} N_{1}}{p^{\alpha-\operatorname{ord} p(N)}}\right) \sum_{a_{2}=0}^{p^{\operatorname{ord} p(N)}-1} 1 \\
& =p^{\operatorname{ord} p(N)} \sum_{\substack{a=1 \\
(a, p)=1}}^{p^{\alpha-\operatorname{ord} p(N)}} \delta_{p}(a) e\left(-\frac{a N_{1}}{p^{\alpha-\operatorname{ord}_{p}(N)}}\right) \\
& =p^{\operatorname{ord}_{p}(N)} V\left(p^{\alpha-\operatorname{ord}_{p}(N)}, N_{1}\right)=p^{\operatorname{ord}_{p}(N)} V\left(p^{\alpha-\operatorname{ord}_{p}(N)}, N p^{-\operatorname{ord}_{p}(N)}\right) \text {. }
\end{aligned}
$$

Таким образом, при $\alpha>\operatorname{ord}_{p}(N) \geqslant 1$ сумма $V\left(p^{\alpha}, N\right)$ рекуррентно выражается через сумму $V\left(p^{v}, N_{1}\right), v=\alpha-\operatorname{ord}_{p}(N), N_{1}=N p^{-\operatorname{ord}_{p}(N)},\left(N_{1}, p\right)=1$. Сумма $V\left(p, N_{1}\right)$ в точности совпадает с суммой Гаусса $S\left(N_{1}, p\right)$, то есть

$$
V\left(p, N_{1}\right)=S\left(N_{1}, p\right)=\delta_{p}\left(N_{1}\right) i^{\left(\frac{p-1}{2}\right)^{2}} p^{\frac{1}{2}}
$$

Поэтому при $\alpha=\operatorname{ord}_{p}(N)+1$ получаем равенство

$$
\begin{aligned}
V\left(p^{\alpha}, N\right) & =V\left(p^{\operatorname{ord}_{p}(N)+1}, N\right)=p^{\operatorname{ord}_{p}(N)} V\left(p, N_{1}\right) \\
& =\delta_{p}\left(N p^{-\operatorname{ord}_{p}(N)}\right) i^{\left(\frac{p-1}{2}\right)^{2}} p^{\operatorname{ord}_{p}(N)+\frac{1}{2}}
\end{aligned}
$$


При $v \geqslant 2$, преобразуя сумму $V\left(p^{v}, N_{1}\right)$ подстановкой $a_{1}+p^{v-1} a_{2}$, где $a_{1}, a_{2}$ независимо пробегают значения $a_{1}=1,2, \ldots, p^{\nu-1}, a_{2}=0,1, \ldots, p-1$, получаем, что

$$
\begin{aligned}
V\left(p^{v}, N_{1}\right) & =\sum_{\substack{a=1 \\
(a, p)=1}}^{p^{\nu}} \delta_{p}(a) e\left(-\frac{a N_{1}}{p^{\nu}}\right) \\
& =\sum_{\substack{a_{1}=1 \\
\left(a_{1}, p\right)=1}}^{p^{\nu-1}} \sum_{a_{2}=1}^{p} \delta_{p}\left(a_{1}+p^{\nu-1} a_{2}\right) e\left(-\frac{N_{1}\left(a_{1}+p^{\nu-1} a_{2}\right)}{p^{\nu}}\right) \\
& =\sum_{\substack{a_{1}=1 \\
\left(a_{1}, p\right)=1}}^{p^{\nu-1}} \delta_{p}\left(a_{1}\right) e\left(-\frac{N a_{1}}{p^{v}}\right) \sum_{a_{2}=1}^{p} e\left(-\frac{N_{1} a_{2}}{p}\right)=0 .
\end{aligned}
$$

Следовательно, при $\alpha \geqslant \operatorname{ord}_{p}(N)+2$

$$
\begin{aligned}
V\left(p^{\alpha}, N\right) & =p^{\operatorname{ord}_{p}(N)} V\left(p^{\alpha-\operatorname{ord}_{p}(N)}, N_{1}\right) \\
& =p^{\operatorname{ord}_{p}(N)} V\left(p^{\alpha-\operatorname{ord}_{p}(N)}, N p^{-\operatorname{ord}_{p}(N)}\right)=0 .
\end{aligned}
$$

Лемма 8. Пусть р-нечетное простое число, $(a, p)=1$, тогда справедлива формула

$$
T_{2}\left(a, p^{\alpha}\right)=\sum_{\substack{n=1 \\(n, p)=1}}^{p^{\alpha}} e\left(\frac{a(n+1)^{2}}{p^{\alpha}}\right)= \begin{cases}S(a, p)-e\left(\frac{a}{p}\right), & \alpha=1 ; \\ p S\left(a, p^{\alpha-2}\right), & \alpha \geqslant 2 .\end{cases}
$$

Доказательство. При $\alpha=1$

$$
\begin{aligned}
T_{2}(a, p) & =\sum_{\substack{n=1 \\
(n, p)=1}}^{p} e\left(\frac{a(n+1)^{2}}{p}\right)=\sum_{n=1}^{p-1} e\left(\frac{a(n+1)^{2}}{p}\right) \\
& =\sum_{n=1}^{p} e\left(\frac{a(n+1)^{2}}{p}\right)-e\left(\frac{a}{p}\right)=\sum_{n=1}^{p} e\left(\frac{a n^{2}}{p}\right)-e\left(\frac{a}{p}\right) \\
& =S(a, p)-e\left(\frac{a}{p}\right) .
\end{aligned}
$$

Пусть теперь $\alpha \geqslant 2$, тогда

$$
\begin{aligned}
& T_{2}\left(a, p^{\alpha}\right)=\sum_{\substack{n=1 \\
(n, p)=1}}^{p^{\alpha}} e\left(\frac{a(n+1)^{2}}{p^{\alpha}}\right) \sum_{\substack{v=0 \\
v \equiv n}}^{p-1} 1 \\
& \left.=\sum_{\substack{v=0 \\
(v, p)=1}}^{p-1} \sum_{\substack{n=1 \\
n \equiv v}}^{p^{\alpha}(\bmod p)} e^{p} p^{\alpha}\right)=\sum_{v=1}^{p-1} T_{v}, \\
& T_{v}=\sum_{n \equiv v}^{p^{\alpha}} e\left(\frac{a(n+1)^{2}}{p^{\alpha}}\right) \text {. }
\end{aligned}
$$


Преобразуя сумму $T_{v}$ подстановкой $y+p^{\alpha-1} z$, где $y, z$ независимо пробегают значения $y=1,2, \ldots, p^{\alpha-1}, z=0,1, \ldots, p-1$, получим, что

$$
\begin{aligned}
T_{v} & =\sum_{\substack{y=1 \\
y \equiv v}}^{p_{(\bmod p)}^{\alpha-1}} \sum_{z=0}^{p-1} e\left(\frac{a\left(y+1+p^{\alpha-1} z\right)^{2}}{p^{\alpha}}\right) \\
& =\sum_{\substack{y=1 \\
y \equiv v}}^{p^{\alpha-1}(\bmod p)}\left(\frac{a(y+1)^{2}}{p^{\alpha}}\right) \sum_{z=0}^{p-1} e\left(\frac{a\left(\left(y+1+p^{\alpha-1} z\right)^{2}-(y+1)^{2}\right)}{p^{\alpha}}\right) .
\end{aligned}
$$

Из соотношения

$$
\begin{aligned}
\left(y+1+p^{\alpha-1} z\right)^{2}-(y+1)^{2} & =2(y+1) p^{\alpha-1} z+p^{2 \alpha-2} z^{2} \\
& \equiv 2(y+1) p^{\alpha-1} z \quad\left(\bmod p^{\alpha}\right)
\end{aligned}
$$

следует, что

$$
\left.T_{v}=\sum_{\substack{y=1 \\ y \equiv v}}^{p^{\alpha-1}(\bmod p)} p^{\alpha}\right) \sum_{z=0}^{p-1} e\left(\frac{2 a(y+1) z}{p}\right) .
$$

Сумма по $z$ является полной линейной тригонометрической суммой по модулю $p$. Поэтому $T_{v}=0$, если $y \not \equiv-1(\bmod p)$, то есть, если $v \neq p-1$. Следовательно, в сумме (15) остается только одно слагаемое с $v=p-1$ :

$$
T_{2}\left(a, p^{\alpha}\right)=T_{p-1}=p \sum_{\substack{y=1 \\ y \equiv p-1}}^{\left.p^{\alpha-1}(\bmod p)\right)} e\left(\frac{a(y+1)^{2}}{p^{\alpha}}\right) .
$$

Отсюда, в частности, при $\alpha=2$, получаем, что

$$
T_{2}\left(a, p^{2}\right)=p \sum_{\substack{y=1 \\ y \equiv p-1(\bmod p)}}^{p} e\left(\frac{a(y+1)^{2}}{p^{2}}\right)=p e\left(\frac{a p^{2}}{p^{2}}\right)=p .
$$

Преобразуя сумму $T_{p-1}$ в (16) подстановкой $y=p-1+p x$, где $x$ пробегает значения $0,1,2, \ldots, p^{\alpha-2}-1$, получаем, что

$$
\begin{aligned}
& \left.T_{p-1}=p \sum_{\substack{y=1 \\
y \equiv p-1}}^{p^{\alpha-1}(\bmod p)} p^{\alpha}\right)=p \sum_{x=0}^{p^{\alpha-2}-1} e\left(\frac{a(y+1)^{2}}{p^{\alpha}}\right) \\
& =p \sum_{x=0}^{p^{\alpha-2}-1} e\left(\frac{a(x+1)^{2}}{p^{\alpha-2}}\right)=p \sum_{x=1}^{p^{\alpha-2}} e\left(\frac{a x^{2}}{p^{\alpha-2}}\right)=p S\left(a, p^{\alpha-2}\right) .
\end{aligned}
$$

Следствие 7. Пусть $p$ - нечетное простое число, $(a, p)=1, x(\alpha)=1$, если $\alpha=1 u$ $\varkappa(\alpha)=0$, если $\alpha \geqslant 2$. Тогда

$$
T\left(a, p^{\alpha}\right)= \begin{cases}\delta_{p}(a) i^{\left(\frac{p-1}{2}\right)^{2}} p^{\frac{\alpha}{2}}-\varkappa(\alpha) e\left(\frac{a}{p}\right), & \text { если } \alpha \text { нечетно } \\ p^{\frac{\alpha}{2}}, & \text { если } \alpha \text { четно. }\end{cases}
$$


Доказательство. Подставляя в правую часть формулы для $T_{2}\left(a, p^{\alpha}\right)$ в лемме 8 точное значение суммы Гаусса из леммы 5 , получаем, что

$$
T(a, p)=S(a, p)-e\left(\frac{a}{p}\right)=\delta_{p}(a) i\left(\frac{p-1}{2}\right)^{2} p^{\frac{1}{2}}-e\left(\frac{a}{p}\right) ;
$$

в случае $\alpha \geqslant 3$ и нечетном $\alpha$

$$
T\left(a, p^{\alpha}\right)=p S\left(a, p^{\alpha-2}\right)=p \delta_{p}(a) i\left(\frac{p-1}{2}\right)^{2} p^{\frac{\alpha-2}{2}}=\delta_{p}(a) i\left(\frac{p-1}{2}\right)^{2} p^{\frac{\alpha}{2}} ;
$$

в случае $\alpha \geqslant 2$ и четном $\alpha$

$$
T\left(a, p^{\alpha}\right)=p S\left(a, p^{\alpha-2}\right)=p \cdot p^{\frac{\alpha-2}{2}}=p^{\frac{\alpha}{2}} .
$$

Лемма 9. Пусть $p$-нечетное простое число, тогда

$$
\begin{aligned}
\Phi(p, N)=\frac{1}{\varphi^{5}(p)}( & -c_{p}(N-5)-10 \varepsilon_{p} p c_{p}(N-3) \\
& \left.\quad-5 p^{2} c_{p}(N-1)+5 p \delta_{p}(N-4)+10 \varepsilon_{p} \delta_{p}(N-2) p^{2}+\delta_{p}(N) p^{3}\right) .
\end{aligned}
$$

Доказательство. Согласно лемме 8, имеет место формула

$$
T_{2}(a, p)=S(a, p)-e\left(\frac{a}{p}\right) .
$$

Поэтому

$$
\begin{aligned}
\Phi(p, N) & =\frac{1}{\varphi^{5}(p)} \sum_{a=1}^{p-1}\left[S(a, p)-e\left(\frac{a}{p}\right)\right]^{5} e\left(-\frac{a N}{p}\right) \\
& =-\frac{1}{\varphi^{5}(p)} \sum_{a=1}^{p-1}\left[1-S(a, p) e\left(-\frac{a}{p}\right)\right]^{5} e\left(\frac{a(5-N)}{p}\right) \\
& =-\frac{1}{\varphi^{5}(p)} \sum_{a=1}^{p-1} \sum_{j=0}^{5}(-1)^{j}\left(\begin{array}{l}
5 \\
j
\end{array}\right) S^{j}(a, p) e\left(\frac{(5-j-N) a}{p}\right) \\
& =\frac{1}{\varphi^{5}(p)} \sum_{j=0}^{5}(-1)^{j+1}\left(\begin{array}{l}
5 \\
j
\end{array}\right) \sum_{a=1}^{p-1} S^{j}(a, p) e\left(\frac{(5-j-N) a}{p}\right) .
\end{aligned}
$$

Пользуясь леммой 5 о точном значении суммы Гаусса для нечетных простых чисел и разбивая сумму по $j$ отдельно по четным и отдельно по нечетным числам, находим, что

$$
\begin{aligned}
\Phi(p, N)= & \frac{1}{\varphi^{5}(p)} \sum_{j=0}^{5}(-1)^{j+1}\left(\begin{array}{l}
5 \\
j
\end{array}\right) i^{\left(\frac{p-1}{2}\right)^{2} j} p^{\frac{j}{2}} \sum_{a=1}^{p-1} \delta_{p}^{j}(a) e\left(\frac{a(5-j-N)}{p}\right) \\
= & \frac{1}{\varphi^{5}(p)}\left(-\sum_{l=0}^{2}\left(\begin{array}{c}
5 \\
2 l
\end{array}\right) p^{l} i^{\left(\frac{p-1}{2}\right)^{2}}{ }^{2 l} \sum_{a=1}^{p-1} e\left(\frac{a(5-2 l-N)}{p}\right)\right. \\
& \left.+\sum_{l=1}^{3}\left(\begin{array}{c}
5 \\
2 l-1
\end{array}\right) p^{l-\frac{1}{2} i} i^{\left(\frac{p-1}{2}\right)^{2}(2 l-1)} \sum_{a=1}^{p-1} \delta_{p}(a) e\left(\frac{a(6-2 l-N)}{p}\right)\right) .
\end{aligned}
$$


В правой части последнего равенства первая сумма по $a$ является суммой Рамануджана $c_{p}(5-2 l-N)$. Вторую сумму по $а$ представим через сумму Гаусса $S(1, p)$, а затем подставим ее значение (лемма 5), в результате получаем, что

$$
\begin{aligned}
\sum_{a=1}^{p-1} \delta_{p}(a) e\left(\frac{(6-2 l-N) a}{p}\right) & =\delta_{p}(6-2 l-N) S(1, p) \\
& =\delta_{p}(6-2 l-N) p^{1 / 2} i^{\left(\frac{p-1}{2}\right)^{2}} .
\end{aligned}
$$

Далее, пользуясь тождеством

$$
i^{\left(\frac{p-1}{2}\right)^{2} 2 l}=(-1)^{\frac{p-1}{2} l},
$$

находим, что

$$
\begin{aligned}
\Phi(p, N)= & \frac{1}{\varphi^{5}(p)}\left(-\sum_{l=0}^{2}\left(\begin{array}{c}
5 \\
2 l
\end{array}\right) p^{l}(-1)^{\frac{p-1}{2} l} c_{p}(5-2 l-N)\right. \\
& \left.+\sum_{l=1}^{3}\left(\begin{array}{c}
5 \\
2 l-1
\end{array}\right) p^{l}(-1)^{\frac{p-1}{2} l} \delta_{p}(6-2 l-N)\right) \\
= & \frac{1}{\varphi^{5}(p)}\left(-\sum_{l=0}^{2}\left(\begin{array}{c}
5 \\
2 l
\end{array}\right) p^{l}(-1)^{\frac{p-1}{2} l} c_{p}(N+2 l-5)\right. \\
& \left.+\sum_{l=1}^{3}\left(\begin{array}{c}
5 \\
2 l-1
\end{array}\right) p^{l}(-1)^{\frac{p-1}{2}(l-1)} \delta_{p}(N+2 l-6)\right) \\
= & \frac{1}{\varphi^{5}(p)}\left(-c_{p}(N-5)-10 \varepsilon_{p} p c_{p}(N-3)-5 p^{2} c_{p}(N-1)\right. \\
& \left.+5 p \delta_{p}(N-4)+10 \varepsilon_{p} \delta_{p}(N-2) p^{2}+\delta_{p}(N) p^{3}\right) .
\end{aligned}
$$

Лемма доказана.

Пользуясь этой леммой соответственно в следствиях 8 и 9, мы вычисляем значения $\Phi(3, N)$ и $\Phi(5, N)$, а в следствии 10 при $p \geqslant 7$ находим оценку снизу для $\Phi(p, N)$.

Следствие 8. Справедлива формула

$$
\Phi(3, N)=\left\{\begin{array}{lll}
2^{-5}, & N \equiv 0 & (\bmod 3) \\
-2^{-4}, & N \equiv 1 & (\bmod 3) \\
2^{-5}, & N \equiv 2 & (\bmod 3)
\end{array}\right.
$$

Доказательство. В утверждении леммы 9, положив $p=3$ и имея в виду, что $\varepsilon_{3}=-1$, находим, что

$$
\begin{gathered}
\Phi(3, N)=2^{-5}\left(-c_{3}(N-5)+30 c_{3}(N-3)-45 c_{3}(N-1)+15 \delta_{3}(N-4)\right. \\
\left.-90 \delta_{3}(N-2)+27 \delta_{3}(N)\right) .
\end{gathered}
$$


Пусть $N \equiv r \quad(\bmod 3), r=0,1,2$, тогда, учитывая, что $\Phi(3, N)=\Phi(3, r)$, получаем, что

$$
\begin{aligned}
\Phi(3,0) & =2^{-5}\left(-c_{3}(1)+30 c_{3}(0)-45 c_{3}(2)+15 \delta_{3}(2)-90 \delta_{3}(1)+27 \delta_{3}(0)\right) \\
& =2^{-5}(1+60+45-15-90)=2^{-5}, \\
\Phi(3,1) & =2^{-5}\left(-c_{3}(2)+30 c_{3}(1)-45 c_{3}(0)+15 \delta_{3}(0)-90 \delta_{3}(2)+27 \delta_{3}(1)\right) \\
& =2^{-5}(1-30-90+90+27)=-2^{-4}, \\
\Phi(3,2) & =2^{-5}\left(-c_{3}(0)+30 c_{3}(2)-45 c_{3}(1)+15 \delta_{3}(1)-90 \delta_{3}(0)+27 \delta_{3}(2)\right) \\
& =2^{-5}(-2-30+45+15-27)=2^{-5} .
\end{aligned}
$$

Следствие 9. Справедлива формула

$$
\Phi(5, N)=\left\{\begin{array}{lll}
-54 \cdot 2^{-10}, & N \equiv 0 & (\bmod 5) \\
99 \cdot 2^{-10}, & N \equiv 1 & (\bmod 5) \\
26 \cdot 2^{-10}, & N \equiv 2 & (\bmod 5) \\
76 \cdot 2^{-10}, & N \equiv 3 & (\bmod 5) \\
51 \cdot 2^{-10}, & N \equiv 4 & (\bmod 5)
\end{array}\right.
$$

Доказательство. Полагая $p=5$ в утверждении леммы 9 и имея в виду, что $\varepsilon_{5}=1$, находим, что

$$
\begin{gathered}
\Phi(5, N)=2^{-10}\left(-c_{5}(N-5)-50 c_{5}(N-3)-125 c_{5}(N-1)\right. \\
\left.+25 \delta_{5}(N-4)+250 \delta_{5}(N-2)+125 \delta_{5}(N)\right) .
\end{gathered}
$$

Пусть $N \equiv r \quad(\bmod 5), r=0,1,2,3,4$, тогда, учитывая, что $\Phi(5, N)=\Phi(5, r)$, получаем, что

$$
\begin{aligned}
\Phi(5,0) & =4^{-5}\left(-c_{5}(0)-50 c_{5}(2)-125 c_{5}(4)+25 \delta_{5}(1)+250 \delta_{5}(3)+125 \delta_{5}(0)\right) \\
& =4^{-5}(-4+50+125+25-250)=-54 \cdot 2^{-10}, \\
\Phi(5,1) & =4^{-5}\left(-c_{5}(1)-50 c_{5}(3)-125 c_{5}(0)+25 \delta_{5}(2)+250 \delta_{5}(4)+125 \delta_{5}(1)\right) \\
& =4^{-5}(1+50-500-25+250+125)=99 \cdot 2^{-10}, \\
\Phi(5,2) & =4^{-5}\left(-c_{5}(2)-50 c_{5}(4)-125 c_{5}(1)+25 \delta_{5}(3)+250 \delta_{5}(0)+125 \delta_{5}(2)\right) \\
& =4^{-5}(1+50+125-25-125)=26 \cdot 2^{-10}, \\
\Phi(5,3) & =4^{-5}\left(-c_{5}(3)-50 c_{5}(0)-125 c_{5}(2)+25 \delta_{5}(4)+250 \delta_{5}(1)+125 \delta_{5}(3)\right) \\
& =4^{-5}(1-200+125+25+250-125)=76 \cdot 2^{-10}, \\
\Phi(5,4) & =4^{-5}\left(-c_{5}(4)-50 c_{5}(1)-125 c_{5}(3)+25 \delta_{5}(0)+250 \delta_{5}(2)+125 \delta_{5}(4)\right) \\
& =4^{-5}(1+50+125-250+125)=51 \cdot 2^{-10} .
\end{aligned}
$$

Следствие 10. При $p \geqslant 7$ справедливы оценки

$$
\begin{array}{ll}
\Phi(p, N)>-\frac{10}{\varphi^{2}(p)}, & (N, p)=1, \\
\Phi(p, N)>-\frac{9}{\varphi^{3}(p)}, & (N, p)=p .
\end{array}
$$


Доказательство. Переходя к оценкам в формуле для $\Phi(p, N)$, полученной в лемме 3.5 , пользуясь точным значением суммы Рамануджана

$$
c_{p}(n)= \begin{cases}-1, & (n, p)=1, \\ p-1, & (n, p)=p,\end{cases}
$$

и имея в виду, что $\left|\varepsilon_{p}\right|=1$, при $(N, p)=1$ находим, что

$$
\begin{aligned}
\Phi(p, N)= & \frac{1}{\varphi^{5}(p)}\left(-c_{p}(N-5)-10 \varepsilon_{p} p c_{p}(N-3)-5 p^{2} c_{p}(N-1)\right. \\
& \left.+5 p \delta_{p}(N-4)+10 \varepsilon_{p} p^{2} \delta_{p}(N-2)+p^{3} \delta_{p}(N)\right) \\
\geqslant & \frac{1}{\varphi^{5}(p)}\left(1-p-10 p(p-1)-5 p^{2}(p-1)-5 p-10 p^{2}-p^{3}\right) \\
= & \frac{1}{\varphi^{5}(p)}\left(1+4 p-15 p^{2}-6 p^{3}\right) \\
= & \frac{1}{\varphi^{5}(p)}\left(-6 p^{2}(p-1)-21 p(p-1)-17(p-1)-16\right) \\
= & \frac{-6 \varphi^{3}(p)-21 \varphi^{2}(p)-17 \varphi(p)-16}{\varphi^{5}(p)} \\
= & -\frac{6}{\varphi^{2}(p)}-\frac{21}{\varphi^{3}(p)}-\frac{17}{\varphi^{4}(p)}-\frac{16}{\varphi^{5}(p)}=-\frac{1}{\varphi^{2}(p)}\left(6+\frac{21}{\varphi(p)}+\frac{17}{\varphi^{2}(p)}+\frac{16}{\varphi^{3}(p)}\right) \\
\geqslant & -\frac{1}{\varphi^{2}(p)}\left(6+\frac{21}{\varphi(7)}+\frac{17}{\varphi^{2}(7)}+\frac{16}{\varphi^{3}(7)}\right)=-\frac{1}{\varphi^{2}(p)}\left(6+\frac{21}{6}+\frac{17}{6^{2}}+\frac{16}{6^{3}}\right) \\
= & -\frac{1}{\varphi^{2}(p)}\left(\frac{19}{2}+\frac{119+16}{7^{2} \cdot 6}\right)=-\frac{1}{\varphi^{2}(p)}\left(\frac{2170}{6^{3}}\right) \\
= & -\frac{1}{\varphi^{2}(p)} \frac{976}{7^{2} \cdot 2}=-\frac{1}{\varphi^{2}(p)} \frac{1085}{113}>-\frac{10}{\varphi^{2}(p)},
\end{aligned}
$$

в то время как для $(N, p)=p$

$$
\begin{aligned}
\Phi(p, N)= & \frac{1}{\varphi^{5}(p)}\left(-c_{p}(N-5)-10 \varepsilon_{p} p c_{p}(N-3)-5 p^{2} c_{p}(N-1)\right. \\
& \left.\quad+5 p \delta_{p}(N-4)+10 \varepsilon_{p} p^{2} \delta_{p}(N-2)+p^{3} \delta_{p}(N)\right) \\
\geqslant & \frac{1}{\varphi^{5}(p)}\left(1-10 p+5 p^{2}-5 p-10 p^{2}\right)=\frac{1}{\varphi^{5}(p)}\left(1-15 p-5 p^{2}\right) \\
= & \frac{1}{\varphi^{5}(p)}(-5 p(p-1)-20(p-1)-21)=\frac{-5 \varphi^{2}(p)-20 \varphi(p)-21}{\varphi^{5}(p)} \\
= & -\frac{5}{\varphi^{3}(p)}-\frac{20}{\varphi^{4}(p)}-\frac{21}{\varphi^{5}(p)}=-\frac{1}{\varphi^{3}(p)}\left(5+\frac{20}{\varphi(p)}+\frac{21}{\varphi^{2}(p)}\right) \\
\geqslant & -\frac{1}{\varphi^{3}(p)}\left(5+\frac{20}{\varphi(7)}+\frac{21}{\varphi^{2}(7)}\right)=-\frac{1}{\varphi^{3}(p)}\left(5+\frac{20}{6}+\frac{21}{6^{2}}\right) \\
= & -\frac{319}{36 \cdot \varphi^{3}(p)}>-\frac{9}{\varphi^{3}(p)} .
\end{aligned}
$$

Следствие доказано. 
Лемма 10. Пусть $p$ - нечетное простое число, $p^{\operatorname{ord}_{p}(N)} \| N, \alpha \geqslant 2$ - четное число. Тогда справедлива формула

$$
\Phi\left(p^{\alpha}, N\right)= \begin{cases}\frac{p^{\frac{5 \alpha}{2}}}{\varphi^{4}\left(p^{\alpha}\right)}, & \alpha \leqslant \operatorname{ord}_{p}(N), \\ -\frac{p^{\frac{7 \text { ord } p(N)+5}{2}}}{\varphi^{5}\left(p^{\operatorname{ord}_{p}(N)+1}\right)}, & \alpha=\operatorname{ord}_{p}(N)+1, \\ 0, & \alpha \geqslant \operatorname{ord}_{p}(N)+2 .\end{cases}
$$

Доказательство. Согласно следствию 7, для четных значений $\alpha$ имеет место формула

$$
T_{2}\left(a, p^{\alpha}\right)=p^{\alpha / 2}
$$

Поэтому

$$
\begin{aligned}
\Phi\left(p^{\alpha}, N\right) & =\frac{1}{\varphi^{5}\left(p^{\alpha}\right)} \sum_{\substack{a=0 \\
(a, p)=1}}^{p^{\alpha}-1} T_{2}^{5}\left(a, p^{\alpha}\right) e\left(-\frac{a N}{p^{\alpha}}\right) \\
& =\frac{p^{\frac{5 \alpha}{2}}}{\varphi^{5}\left(p^{\alpha}\right)} \sum_{\substack{a=1 \\
(a, p)=1}}^{p^{\alpha}} e\left(-\frac{a N}{p^{\alpha}}\right)=\frac{p^{\frac{5 \alpha}{2}}}{\varphi^{5}\left(p^{\alpha}\right)} c_{p^{\alpha}}(-N),
\end{aligned}
$$

где $c_{p^{\alpha}}(-N)-$ сумма Рамануджана. Если $\alpha \leqslant \operatorname{ord}_{p}(N)$, то

$$
c_{p^{\alpha}}(-N)=\varphi\left(p^{\alpha}\right) .
$$

Поэтому

$$
\Phi\left(p^{\alpha}, N\right)=\frac{p^{5 \alpha / 2}}{\varphi^{4}\left(p^{\alpha}\right)} .
$$

Если $\alpha \geqslant \operatorname{ord}_{p}(N)+1$, то согласно лемме 6

$$
c_{p^{\alpha}}(N)=p^{\operatorname{ord}_{p}(N)} \mu\left(p^{\alpha-\operatorname{ord}_{p}(N)}\right) .
$$

Следовательно,

$$
\begin{aligned}
\Phi\left(p^{\alpha}, N\right) & =\frac{p^{\frac{5 \alpha}{2}}}{\varphi^{5}\left(p^{\alpha}\right)} c_{p^{\alpha}}(-N)=\frac{p^{\frac{5 \alpha}{2}}}{\varphi^{5}\left(p^{\alpha}\right)} p^{\operatorname{ord}_{p}(N)} \mu\left(p^{\alpha-\operatorname{ord}_{p}(N)}\right) \\
& =\frac{p^{\frac{5 \alpha}{2}+\operatorname{ord}_{p}(N)}}{\varphi^{5}\left(p^{\alpha}\right)} \mu\left(p^{\alpha-\operatorname{ord}_{p}(N)}\right) .
\end{aligned}
$$

Лемма доказана.

Лемма 11. Пусть $p$ - нечетное простое число, $p^{\operatorname{ord}_{p}(N)} \| N, \alpha \geqslant 3$ - нечетное число. Тогда справедлива формула

$$
\Phi\left(p^{\alpha}, N\right)= \begin{cases}(-1)^{\frac{p-1}{2}} \delta_{p}\left(N p^{-\operatorname{ord}_{p}(N)}\right) \frac{p^{\frac{7 \text { ord } p(N)}{2}+3}}{\varphi^{5}\left(p^{\operatorname{ord}_{p}(N)+1}\right)}, & \alpha=\operatorname{ord}_{p}(N)+1, \\ 0, & \alpha \neq \operatorname{ord}_{p}(N)+1,\end{cases}
$$


Доказательство. Пользуясь следствием 7 для нечетных $\alpha \geqslant 3$, находим, что

$$
\begin{aligned}
\Phi\left(p^{\alpha}, N\right) & =\frac{1}{\varphi^{5}\left(p^{\alpha}\right)} \sum_{\substack{a=0 \\
(a, p)=1}}^{p^{\alpha}-1} T_{2}^{5}\left(a, p^{\alpha}\right) e\left(-\frac{a N}{p^{\alpha}}\right) \\
& =\frac{1}{\varphi^{5}\left(p^{\alpha}\right)} \sum_{\substack{a=1 \\
(a, p)=1}}^{p^{\alpha}}\left[\delta_{p}(a) i\left(\frac{p-1}{2}\right)^{2} p^{\frac{\alpha}{2}}\right]^{5} e\left(-\frac{a N}{p^{\alpha}}\right) \\
& =\frac{i^{\left(\frac{p-1}{2}\right)^{2}} p^{\frac{5 \alpha}{2}}}{\varphi^{5}\left(p^{\alpha}\right)} \sum_{\substack{a=1 \\
(a, p)=1}}^{p^{\alpha}} \delta_{p}(a) e\left(-\frac{a N}{p^{\alpha}}\right)=\frac{i^{\left(\frac{p-1}{2}\right)^{2} p^{\frac{5 \alpha}{2}}}}{\varphi^{5}\left(p^{\alpha}\right)} V\left(p^{\alpha}, N\right) .
\end{aligned}
$$

Подставляя при $\alpha=\operatorname{ord}_{p}(N)+1$ в правую часть равенства значение $V\left(p^{\operatorname{ord}_{p}(N)+1}, N\right)$ из леммы 4 и имея в виду, что

$$
i^{\left(\frac{p-1}{2}\right)^{2} \cdot 2}=(-1)^{\left(\frac{p-1}{2}\right)^{2}}=(-1)^{\frac{p-1}{2}},
$$

находим, что

$$
\begin{aligned}
\Phi\left(p^{\operatorname{ord} p(N)+1}, N\right) & =\frac{i^{\left(\frac{p-1}{2}\right)^{2}} p^{\frac{5(\operatorname{ord} p(N)+1)}{2}}}{\varphi^{5}\left(p^{\operatorname{ord} p(N)+1}\right)} \delta_{p}\left(N p^{-\operatorname{ord}_{p}(N)}\right) i\left(\frac{p-1}{2}\right)^{2} p^{\operatorname{ord} p(N)+\frac{1}{2}} \\
& =(-1)^{\frac{p-1}{2}} \delta_{p}\left(N p^{-\operatorname{ord}_{p}(N)}\right) \frac{p^{\frac{7 \operatorname{ord} p(N)}{2}+3}}{\varphi^{5}\left(p^{\operatorname{ord} p(N)+1}\right)} .
\end{aligned}
$$

Если $\alpha \neq \operatorname{ord}_{p}(N)+1$, то, согласно лемме $4, V\left(p^{\alpha}, N\right)=0$, поэтому $\Phi\left(p^{\alpha}, N\right)=0$.

Следствие 11. Пусть $p$ - нечетное простое число, $(N, p)=1, \alpha \geqslant 2$, тогда $\Phi\left(p^{\alpha}, N\right)=0$.

Это утверждение непосредственно следует из лемм 11 и 10 при $\operatorname{ord}_{p}(N)=0$.

Доказательство теоремьл 3. Пусть $\operatorname{ord}_{p}(N)=\beta$. Справедливы равенства

$$
\begin{aligned}
\mathscr{F}_{(}(p, N) & =1+\Phi(p, N)+\mathscr{F}_{1}(p, N), \\
\mathscr{F}_{1}(p, N) & =\sum_{\alpha=2}^{\infty} \Phi\left(p^{\alpha}, N\right) .
\end{aligned}
$$

Значение $\Phi(p, N)$ вычислено в лемме 9. Для вычисления $\mathscr{F}_{1}(p, N)$ рассмотрим три возможных случая.

(i) $\beta=0$. Согласно следствию 11, для всех $\alpha \geqslant 2$ верно равенство $\Phi\left(p^{\alpha}, N\right)=0$, поэтому $\mathscr{F}_{1}(p, N)=0$. 
(ii) $\beta \geqslant 1-$ нечетное число. $\quad$ В этом случае для четных $\alpha \geqslant \beta+3$ из леммы 10 , a для нечетных $\alpha \geqslant 3$ из леммы 11 , соответственно, следует, что $\Phi\left(p^{\alpha}, N\right)=0$, а значение $\Phi\left(p^{\alpha}, N\right)$ для четных $\alpha, 2 \leqslant \alpha \leqslant \beta+1$ определяется леммой 10 . Поэтому

$$
\begin{aligned}
& \mathscr{F}_{1}(p, N)=\sum_{\alpha \equiv 0}^{\beta-1} \Phi\left(p^{\alpha}, N\right)+\Phi\left(p^{\beta+1}, N\right)=\sum_{\substack{\alpha=0 \\
\alpha=2}}^{\beta-1} \frac{p^{\frac{5 \alpha}{2}}}{\varphi^{4}\left(p^{\alpha}\right)}-\frac{p^{\frac{7 \beta+5}{2}}}{\varphi^{5}\left(p^{\beta+1}\right)} \\
& =\sum_{\alpha \equiv 0}^{\beta-1} \frac{p^{\frac{5 \alpha}{2}}}{\left(p^{\alpha-1}(p-1)\right)^{4}}-\frac{p^{\frac{7 \beta+5}{2}}}{\left(p^{\beta}(p-1)\right)^{5}} \\
& =\frac{p^{4}}{(p-1)^{4}} \sum_{\alpha=0}^{\beta-1} p^{-\frac{3}{2} \alpha}-\frac{p^{-\frac{3}{2} \beta+\frac{5}{2}}}{(p-1)^{5}} \\
& =\frac{p^{4}}{(p-1)^{4}} \sum_{\alpha_{1}=1}^{\frac{1}{2}(\beta-1)} p^{-3 \alpha_{1}}-\frac{p^{-\frac{3}{2} \beta+\frac{5}{2}}}{(p-1)^{5}}=\frac{p}{(p-1)^{4}} \sum_{\alpha_{1}=0}^{\frac{1}{2}(\beta-1)-1} p^{-3 \alpha_{1}}-\frac{p^{-\frac{3}{2} \beta+\frac{5}{2}}}{(p-1)^{5}} \\
& =\frac{p}{(p-1)^{4}} \frac{1-p^{-\frac{3}{2}(\beta-1)}}{1-p^{-3}}-\frac{p^{-\frac{3}{2}(\beta-1)+1}}{(p-1)^{5}} \\
& =-\frac{p}{(p-1)^{5}}+\frac{p}{(p-1)^{4}}\left(\frac{1-p^{-\frac{3}{2}(\beta-1)}}{1-p^{-3}}+\frac{1-p^{-\frac{3}{2}(\beta-1)}}{p-1}\right) \\
& =-\frac{p}{(p-1)^{5}}+\frac{p\left(1-p^{-\frac{3}{2}(\beta-1)}\right)}{(p-1)^{4}}\left(\frac{1}{1-p^{-3}}+\frac{1}{p-1}\right) \\
& =-\frac{p}{(p-1)^{5}}+\frac{p^{5}-p}{(p-1)^{5}\left(p^{3}-1\right)}\left(1-p^{-\frac{3}{2}(\beta-1)}\right)=-\frac{p}{(p-1)^{5}}+\Delta(p, N) \text {, } \\
& \Delta(p, N)=\frac{p^{5}-p}{(p-1)^{5}\left(p^{3}-1\right)}\left(1-\frac{1}{p^{\frac{3}{2}(\beta-1)}}\right) \text {. }
\end{aligned}
$$

(iii) $\beta \geqslant 2-$ четное число. Соответственно, для четных $\alpha \geqslant \beta+2$ из леммы 10 и для нечетных $\alpha \neq \beta+1, \alpha \geqslant 3$, из леммы 11 следует, что $\Phi\left(p^{\alpha}, N\right)=0$. Определяя $\Phi\left(p^{\alpha}, N\right)$ при четных $2 \leqslant \alpha \leqslant \beta$ леммой 10 , а $\Phi\left(p^{\beta+1}, N\right)$ - леммой 11 , находим, что

$$
\begin{aligned}
\mathscr{F}_{1}(p, N) & =\sum_{\alpha \equiv 0}^{\beta=2} \Phi\left(p^{\alpha}, N\right)+\Phi\left(p^{\beta+1}, N\right) \\
& =\sum_{\alpha \equiv 0}^{\beta=2} \frac{p^{\frac{5 \alpha}{2}}}{\varphi^{4}\left(p^{\alpha}\right)}+\varepsilon_{p} \delta_{p}(N) \frac{p^{\frac{7 \beta}{2}+3}}{\varphi^{5}\left(p^{\beta+1}\right)} \\
& =\sum_{\alpha \equiv 0}^{\beta=2} \frac{p^{\frac{5 \alpha}{2}}}{\left(p^{\alpha-1}(p-1)\right)^{4}}+\varepsilon_{p} \delta_{p}(N) \frac{p^{\frac{7 \beta}{2}+3}}{\left(p^{\beta}(p-1)\right)^{5}}
\end{aligned}
$$




$$
\begin{aligned}
& =\frac{p^{4}}{(p-1)^{4}} \sum_{\substack{\alpha=2 \\
\alpha=0}}^{\beta} p^{-\frac{3}{2} \alpha}+\varepsilon_{p} \delta_{p}(N) \frac{p^{-\frac{3}{2} \beta+3}}{(p-1)^{5}} \\
& =\frac{p^{4}}{(p-1)^{4}} \sum_{\alpha_{1}=1}^{\frac{1}{2} \beta} p^{-3 \alpha_{1}}+\varepsilon_{p} \delta_{p}(N) \frac{p^{-\frac{3}{2} \beta+3}}{(p-1)^{5}} \\
& =\frac{p}{(p-1)^{4}} \sum_{\alpha_{1}=0}^{\frac{1}{2} \beta-1} p^{-3 \alpha_{1}}+\varepsilon_{p} \delta_{p}(N) \frac{p^{-\frac{3}{2} \beta+3}}{(p-1)^{5}} \\
& =\frac{p}{(p-1)^{4}}\left(\frac{1-p^{-\frac{3}{2} \beta}}{1-p^{-3}}+\varepsilon_{p} \delta_{p}(N) \frac{p^{-\frac{3}{2} \beta+2}}{p-1}\right) \\
& =\frac{p}{(p-1)^{4}}\left(\frac{1-p^{-\frac{3}{2} \beta}}{1-p^{-3}}-\frac{p^{-\frac{3}{2} \beta+2}}{p-1}+\left(1+\varepsilon_{p} \delta_{p}(N)\right) \frac{p^{-\frac{3}{2} \beta+2}}{p-1}\right) \\
& =\frac{p}{(p-1)^{4}}\left(\frac{1-p^{-\frac{3}{2} \beta}}{1-p^{-3}}-\frac{p^{-\frac{3}{2} \beta+2}}{p-1}\right)+\Delta(p, N) \\
& =\frac{p}{(p-1)^{4}}\left(\frac{1-p^{-\frac{3}{2} \beta}}{1-p^{-3}}-\frac{p^{-\frac{3}{2} \beta+1}\left(1+p^{-1}+p^{-2}\right)}{1-p^{-3}}\right)+\Delta(p, N) \\
& =\frac{p}{(p-1)^{4}\left(1-p^{-3}\right)}\left(1-p^{-\frac{3}{2}(\beta-2)-2}\left(1+2 p^{-1}+p^{-2}\right)\right)+\Delta(p, N) \\
& =\frac{p^{4}}{(p-1)^{4}\left(p^{3}-1\right)}\left(1-\frac{(p+1)^{2}}{p^{\frac{3}{2}(\beta-2)+4}}\right)+\Delta(p, N), \\
& \Delta(p, N)=\frac{1+\varepsilon_{p} \delta_{p}(N)}{(p-1)^{5} p^{\frac{3}{2}(\beta-2)}} .
\end{aligned}
$$

Доказательство следствия 1. Рассмотрим три возможных случая.

(I) $\beta=0 . \quad$ Из теоремы 3 и следствия 10, соответственно, следует, что

$$
\mathscr{F}(p, N)=1+\Phi(p, N), \quad \Phi(p, N)>-\frac{10}{\varphi^{2}(p)} .
$$

Следовательно,

$$
\mathscr{F}(p, N)>1-\frac{10}{\varphi^{2}(p)}
$$

(II) $\beta \geqslant 1$ нечетно. $\mathrm{B}$ этом случае, также пользуясь следствием 10 и теоремой 3 , находим, что

$$
\begin{aligned}
\Phi(p, N) & >-\frac{9}{\varphi^{3}(p)}, \\
\mathscr{F}_{1}(p, N) & =-\frac{p}{(p-1)^{5}}+\frac{p^{5}-p}{(p-1)^{5}\left(p^{3}-1\right)}\left(1-\frac{1}{p^{\frac{3}{2}(\beta-1)}}\right) \\
& \geqslant-\frac{p}{(p-1)^{5}}=-\frac{1}{\varphi^{3}(p)} \frac{p}{(p-1)^{2}} \geqslant-\frac{1}{\varphi^{3}(p)} .
\end{aligned}
$$


Поэтому

$$
\begin{aligned}
\mathscr{F}(p, N) & =1+\Phi(p, N)+\mathscr{F}_{1}(p, N) \\
& >1-\frac{10}{\varphi^{3}(p)}>1-\frac{10}{\varphi^{2}(p)} .
\end{aligned}
$$

(III) $\beta \geqslant 2$ четно. Поступая, как в предыдущих случаях, то есть применяя следствие 10 и теорему 3 , находим, что

$$
\begin{aligned}
\Phi(p, N) & >-\frac{9}{\varphi^{3}(p)} \\
\mathscr{F}_{1}(p, N) & =\frac{p^{4}}{(p-1)^{4}\left(p^{3}-1\right)}\left(1-\frac{(p+1)^{2}}{p^{\frac{3}{2}(\beta-2)+4}}\right)+\frac{1+\varepsilon_{p} \delta_{p}(N)}{(p-1)^{5} p^{\frac{3}{2}(\beta-2)}} \\
& \geqslant \frac{p^{4}}{(p-1)^{4}\left(p^{3}-1\right)}\left(1-\frac{(p+1)^{2}}{p^{\frac{3}{2}(\beta-2)+4}}\right) \\
& \geqslant \frac{p^{4}}{(p-1)^{4}\left(p^{3}-1\right)}\left(1-\frac{(p+1)^{2}}{p^{4}}\right) \\
& >0>-\frac{1}{\varphi^{3}(p)}
\end{aligned}
$$

и поэтому

$$
\begin{aligned}
\mathscr{F}(p, N) & =1+\Phi(p, N)+\mathscr{F}_{1}(p, N) \\
& >1-\frac{10}{\varphi^{3}(p)}>1-\frac{10}{\varphi^{2}(p)} .
\end{aligned}
$$

Следствие доказано.

Доказательство следствия 3. Полагая $p=3$ в утверждении теоремы 3, находим, что

$$
\mathscr{F}_{1}(3, N)= \begin{cases}0, & \text { если } \beta=0, \\ -\frac{3}{32}+\frac{15}{52}\left(1-\frac{1}{3^{\frac{3}{2}(\beta-1)}}\right), & \text { если } \beta \geqslant 1 \text { нечетно, } \\ \frac{3^{4}}{2^{4} \cdot 26}\left(1-\frac{2^{4}}{3^{\frac{3}{2}(\beta-2)+4}}\right)+\frac{1-\delta_{3}(N)}{2^{5} \cdot 3^{\frac{3}{2}(\beta-2)},} & \text { если } \beta \geqslant 2 \text { четно. }\end{cases}
$$

Согласно следствию 8,

$$
\Phi(3, N)=\left\{\begin{array}{lll}
2^{-5}, & \text { если } \beta \geqslant 1, & \\
-2^{-4}, & \text { если } N \equiv 1 & (\bmod 3) \\
2^{-5}, & \text { если } N \equiv 2 & (\bmod 3)
\end{array}\right.
$$

Подставляя эти значения в формулу

$$
\mathscr{F}(3, N)=1+\Phi(3, N)+\mathscr{F}_{1}(3, N),
$$

получим утверждение следствия.

Следствие 4 непосредственно вытекает из следствия 3. 
Доказательство следствия 5. Полагая $p=5$ в утверждении теоремы 3 , находим, что

$$
\mathscr{F}_{1}(5, N)= \begin{cases}0, & \text { если } \beta=0, \\ -\frac{5}{2^{10}}+\frac{5^{4}}{31 \cdot 2^{10}}\left(1-\frac{1}{5^{\frac{3}{2}(\beta-1)}}\right), & \text { если } \beta \geqslant 1 \text { нечетно, } \\ \frac{5^{4}}{31 \cdot 2^{10}}-\frac{3^{2}}{31 \cdot 2^{8} \cdot 5^{\frac{3}{2}(\beta-2)}}+\frac{1+\delta_{5}(N)}{2^{10} \cdot 5^{\frac{3}{2}(\beta-2)}}, & \text { если } \beta \geqslant 2 \text { четно. }\end{cases}
$$

Согласно следствию 9

$$
\Phi(5, N)=\left\{\begin{array}{lll}
-54 \cdot 2^{-10}, & \text { если } N \equiv 0 & (\bmod 5) \\
99 \cdot 2^{-10}, & \text { если } N \equiv 1 & (\bmod 5) \\
26 \cdot 2^{-10}, & \text { если } N \equiv 2 & (\bmod 5) \\
76 \cdot 2^{-10}, & \text { если } N \equiv 3 & (\bmod 5) \\
51 \cdot 2^{-10}, & \text { если } N \equiv 4 & (\bmod 5)
\end{array}\right.
$$

Подставляя эти значения в формулу

$$
\mathscr{F}(5, N)=1+\Phi(5, N)+\mathscr{F}_{1}(5, N),
$$

получаем утверждение следствия.

Доказательство следствия 6. Утверждение непосредственно вытекает из следствия 5 с учетом неравенства

$$
\begin{aligned}
\mathscr{F}(5, N) & \geqslant 1-\frac{1049}{31 \cdot 2^{10}}-\frac{3^{2}}{31 \cdot 2^{8} \cdot 5^{\frac{3}{2}(\beta-2)}}+\frac{1+\delta_{5}(N)}{2^{10} \cdot 5^{\frac{3}{2}(\beta-2)}} \\
& \geqslant 1-\frac{1049}{31 \cdot 2^{10}}-\frac{3^{2}}{31 \cdot 2^{8} \cdot 5^{\frac{3}{2}(\beta-2)}} \\
& \geqslant 1-\frac{1049}{31 \cdot 2^{10}}-\frac{3^{2}}{31 \cdot 2^{8}}=1-\frac{1049+36}{31 \cdot 2^{10}} \\
& =1-\frac{35}{2^{10}} \geqslant 1-\frac{59}{2^{10}} .
\end{aligned}
$$

\section{4. Вычисление $\subseteq(N)$ и ее оценка снизу}

В этом параграфе докажем основную теорему 1.

Лемма 12. Сумма $T_{2}(a, q),(a, q)=1-$ мультипликативная функция, то есть если $q=q_{1} q_{2} u\left(q_{1}, q_{2}\right)=1$, mo

$$
T_{2}(a, q)=T_{2}\left(a_{1}, q_{1}\right) T_{2}\left(a_{2}, q_{2}\right),
$$

где $a_{1}, a_{2}$ однозначно определяются из сравнения

$$
a \equiv a_{1} q_{2}+a_{2} q_{1} \quad\left(\bmod q_{1} q_{2}\right)
$$

и условий $1 \leqslant a_{1} \leqslant q_{1}, 1 \leqslant a_{2} \leqslant q_{2}$. 
Доказательство. Любой вычет $n$ по модулю $q_{1} q_{2}$ можно представить единственным способом в виде

$$
n \equiv n_{1} q_{2}+n_{2} q_{1} \quad\left(\bmod q_{1} q_{2}\right), \quad 1 \leqslant n_{1} \leqslant q_{1}, \quad 1 \leqslant n_{2} \leqslant q_{2} .
$$

Пользуясь этим представлением, получаем, что

$$
\begin{aligned}
(n, q) & =\left(n_{1} q_{2}+n_{2} q_{1}, q_{1} q_{2}\right)=\left(n_{1} q_{2}, q_{1}\right)\left(n_{2} q_{1}, q_{2}\right) \\
& =\left(n_{1} q_{2}, q_{1}\right)\left(n_{2} q_{1}, q_{2}\right)=\left(n_{1}, q_{1}\right)\left(n_{2}, q_{2}\right) .
\end{aligned}
$$

Точно так же любой вычет $a$ по модулю $q_{1} q_{2}$ можно представить единственным способом в виде

$$
a \equiv a_{1} q_{2}+a_{2} q_{1} \quad\left(\bmod q_{1} q_{2}\right), \quad 1 \leqslant a_{1} \leqslant q_{1}, \quad 1 \leqslant a_{2} \leqslant q_{2},
$$

и

$$
(n, q)=\left(n_{1}, q_{1}\right)\left(n_{2}, q_{2}\right)
$$

Поэтому

$$
\begin{aligned}
T_{2}(a, q) & =T_{2}\left(a_{1} q_{2}+a_{2} q_{1}, q_{1} q_{2}\right) \\
& =\sum_{\substack{n_{1}=1 \\
\left(n_{1}, q_{1}\right)=1}}^{q_{1}} \sum_{\substack{n_{2}=1 \\
\left(n_{2}, q_{2}\right)=1}}^{q_{2}} e\left(\frac{\left(a_{1} q_{2}+a_{2} q_{1}\right)\left(n_{1} q_{2}+n_{2} q_{1}+1\right)^{2}}{q_{1} q_{2}}\right) \\
& \left.=\sum_{\substack{n_{1}=1 \\
\left(n_{1}, q_{1}\right)=1}}^{q_{1}} \sum^{q_{1}}\right) \sum_{\substack{n_{2}=1 \\
\left(n_{2}, q_{2}\right)=1}}^{q_{2}} e\left(\frac{a_{2}\left(n_{2} q_{1}+1\right)^{2}}{q_{2}}\right) \\
& =T_{2}\left(a_{1}, q_{1}\right) T_{2}\left(a_{2}, q_{2}\right) .
\end{aligned}
$$

Лемма 13. Для суммы $T_{2}(a, q),(a, q)=1$, справедлива оценка

$$
T_{2}(a, q)<\sqrt{2} e^{2 \sqrt{2 \ln q}} \sqrt{q} .
$$

Доказательство. Пусть

$$
q=2^{\alpha_{0}} p_{1}^{\alpha_{1}} \cdots p_{k}^{\alpha_{k}}
$$

- каноническое разложение числа $q$ на простые сомножители, тогда в силу мультипликативности $T_{2}(a, q)$ и однозначности разложения натуральных чисел на простые сомножители

$$
T_{2}(a, q)=T_{2}\left(a_{0}, 2^{\alpha_{0}}\right) T_{2}\left(a_{1}, p^{\alpha_{1}}\right) \cdots T_{2}\left(a_{k}, p^{\alpha_{k}}\right) .
$$

Пользуясь леммой 2 и следствием 7 , для $T_{2}\left(a_{0}, 2^{\alpha}\right)$ и $T_{2}\left(a_{1}, p^{\alpha_{1}}\right)$, соответственно, находим оценки

$$
\begin{aligned}
& \left|T_{2}\left(a_{0}, 2^{\alpha_{0}}\right)\right| \leqslant 2^{\frac{\alpha_{0}+1}{2}}, \\
& \left|T_{2}\left(a_{i}, p^{\alpha_{i}}\right)\right| \leqslant p^{\frac{\alpha}{2}}+\varkappa(\alpha), \quad 1 \leqslant i \leqslant k,
\end{aligned}
$$


где $\varkappa(\alpha)=1$, если $\alpha=1$ и $\varkappa(\alpha)=0$, если $\alpha \geqslant 2$. Поэтому

$$
\begin{aligned}
\left|T_{2}(a, q)\right| & =\left|T_{2}\left(a_{0}, 2_{0}^{\alpha}\right)\right|\left|T_{2}\left(a_{1}, p^{\alpha_{1}}\right)\right| \cdots\left|T_{2}\left(a_{k}, p^{\alpha_{k}}\right)\right| \\
& \leqslant 2^{\frac{\alpha+1}{2}}\left(p_{1}^{\frac{\alpha_{1}}{2}}+\varkappa\left(\alpha_{1}\right)\right) \cdots\left(p_{k}^{\frac{\alpha_{k}}{2}}+\varkappa\left(\alpha_{k}\right)\right) \leqslant m(q) \sqrt{2 q}, \\
m(q) & =\left(1+\frac{\varkappa\left(\alpha_{1}\right)}{p_{1}^{\frac{\alpha_{1}}{2}}}\right)\left(1+\frac{\varkappa\left(\alpha_{2}\right)}{p_{2}^{\frac{\alpha_{2}}{2}}}\right) \cdots\left(1+\frac{\varkappa\left(\alpha_{k}\right)}{p_{k}^{\frac{\alpha_{k}}{2}}}\right) .
\end{aligned}
$$

Пусть $q_{i}-i$-е простое число; очевидно, существует $k$ такое, что

$$
q^{\prime}=q_{1} q_{2} \cdots q_{k} \leqslant q<q_{1} q_{2} \cdots q_{k} q_{k+1}, \quad k \geqslant 1 .
$$

Согласно закону распределения простых чисел,

$$
\ln q^{\prime}=\sum_{i \leqslant k} \ln q_{i}=\sum_{p \leqslant q_{k}} \ln p \geqslant \frac{q_{k}}{2},
$$

так что

$$
q_{k} \leqslant 2 \ln q^{\prime} \leqslant 2 \ln q
$$

Из определения числа $q_{k}$, очевидно, следует, что

$$
\begin{aligned}
m(q) & \leqslant\left(1+\frac{1}{\sqrt{q_{1}}}\right)\left(1+\frac{1}{\sqrt{q_{2}}}\right) \cdots\left(1+\frac{1}{\sqrt{q_{k}}}\right) \\
& \leqslant \prod_{p \leqslant 2 \ln q}\left(1+\frac{1}{\sqrt{p}}\right) .
\end{aligned}
$$

Логарифмируя последнее неравенство и пользуясь разложением функции $\ln (1+x)$, находим, что

$$
\begin{aligned}
\ln m(q) & \leqslant \sum_{p \leqslant 2 \ln q} \ln \left(1+\frac{1}{\sqrt{p}}\right) \leqslant \sum_{p \leqslant 2 \ln q} \frac{1}{\sqrt{p}} \leqslant \sum_{n \leqslant 2 \ln q} \frac{1}{\sqrt{n}} \\
& \leqslant 1+\int_{1}^{2 \ln q} \frac{d u}{\sqrt{u}}=1+2 \sqrt{2 \ln q}-2<2 \sqrt{2 \ln q} .
\end{aligned}
$$

Доказательство теоремь 1. Справедливы равенства

$$
\begin{aligned}
\Im & =\Im(N)=\sum_{q=1}^{\infty} \Phi(q, N), \\
\Phi(q, N) & =\frac{1}{\varphi^{5}(q)} \sum_{\substack{a=0 \\
(a, q)=1}}^{q-1} T_{2}^{5}(a, q) e\left(-\frac{a N}{q}\right) .
\end{aligned}
$$

Покажем, что сумма $\Phi(q, N)$ является мультипликативной функцией. Пусть $q=q_{1} q_{2}$, $\left(q_{1}, q_{2}\right)=1$. Любой вычет $n$ по модулю $q_{1} q_{2}$ можно представить единственным способом в виде

$$
n \equiv n_{1} q_{2}+n_{2} q_{1} \quad\left(\bmod q_{1} q_{2}\right), \quad 1 \leqslant n_{1} \leqslant q_{1}, \quad 1 \leqslant n_{2} \leqslant q_{2} .
$$


Пользуясь этим представлением, получаем, что

$$
(n, q)=\left(n_{1}, q_{1}\right)\left(n_{2}, q_{2}\right) .
$$

Точно так же любой вычет $a$ по модулю $q_{1} q_{2}$ можно представить единственным способом в виде

$$
a \equiv a_{1} q_{2}+a_{2} q_{1} \quad\left(\bmod q_{1} q_{2}\right), \quad 1 \leqslant a_{1} \leqslant q_{1}, \quad 1 \leqslant a_{2} \leqslant q_{2},
$$

и

$$
(a, q)=\left(a_{1}, q_{1}\right)\left(a_{2}, q_{2}\right)
$$

Поэтому, согласно лемме 12,

$$
T_{2}(a, q)=T_{2}\left(a_{1}, q_{1}\right) T_{2}\left(a_{2}, q_{2}\right) .
$$

Следовательно,

$$
\begin{aligned}
\Phi(q, N) & =\Phi\left(q_{1} q_{2}, N\right) \\
& =\frac{1}{\varphi^{5}\left(q_{1} q_{2}\right)} \sum_{\substack{a_{1}=1 \\
\left(a_{1}, q_{1}\right)=1}}^{q_{1}} \sum_{\substack{a_{2}=1 \\
\left(a_{2}, q_{2}\right)=1}}^{q_{2}} T_{2}^{5}\left(a_{1}, q_{1}\right) T_{2}^{5}\left(a_{2}, q_{2}\right) e\left(-\frac{\left(a_{1} q_{2}+a_{2} q_{1}\right) N}{q_{1} q_{2}}\right) \\
& =\frac{1}{\varphi^{5}\left(q_{1}\right)} \sum_{\substack{a_{1}=1 \\
\left(a_{1}, q_{1}\right)=1}}^{q_{1}} T_{2}^{5}\left(a_{1}, q_{1}\right) e\left(-\frac{a_{1} N}{q_{1}}\right) \frac{1}{\varphi^{5}\left(q_{2}\right)} \sum_{\substack{a_{2}=1 \\
\left(a_{2}, q_{2}\right)=1}}^{q_{2}} T_{2}^{5}\left(a_{2}, q_{2}\right) e\left(-\frac{a_{2} N}{q_{2}}\right) \\
& =\Phi\left(q_{1}, N\right) \Phi\left(q_{2}, N\right) .
\end{aligned}
$$

При целом $X \geqslant 2$, в силу мультипликативности $\Phi(q, N)$ и однозначности разложения натуральных чисел на простые сомножители,

$$
\begin{aligned}
\prod_{p \leqslant X}\left(1+\Phi(p, N)+\Phi\left(p^{2}, N\right)+\cdots\right) & =\sum_{q \leqslant X} \Phi(q, N)+R(X), \\
R(X) & =\sum_{q>X}^{\star} \Phi(q, N),
\end{aligned}
$$

где звездочка означает, что суммирование ведется по всем натуральным числам $q$, имеющим только простые делители $p, p \leqslant X$. Применяя лемму 13, находим, что

$$
\begin{aligned}
|\Phi(q, N)| & =\left|\frac{1}{\varphi^{5}(q)} \sum_{\substack{a=0 \\
(a, q)=1}}^{q-1} T_{2}^{5}(a, q) e\left(-\frac{a N}{q}\right)\right| \\
& <\frac{\left(\sqrt{2} e^{2 \sqrt{2 \ln q}} \sqrt{q}\right)^{5}}{\varphi^{4}(q)}=\frac{2^{\frac{5}{2}} e^{10 \sqrt{2 \ln q}}}{q^{3 / 2}}\left(\frac{q}{\varphi(q)}\right)^{4} \ll q^{-\frac{3}{2}+\varepsilon} .
\end{aligned}
$$

Далее, переходя в $R(X)$ к оценкам, и затем подставляя полученную оценку для $\Phi(q, N)$, находим, что

$$
|R(X)| \ll \sum_{q>X} q^{-\frac{3}{2}+\varepsilon} \leqslant \int_{X}^{\infty} u^{-\frac{3}{2}+\varepsilon} d u \ll X^{-\frac{1}{2}+\varepsilon}
$$


Следовательно, соотношение (17) принимает вид

$$
\prod_{p \leqslant X}\left(1+\Phi(p, N)+\Phi\left(p^{2}, N\right)+\cdots\right)=\sum_{q \leqslant X} \Phi(q, N)+O\left(X^{-\frac{1}{2}+\varepsilon}\right) .
$$

Переходя к пределу при $X \rightarrow \infty$ в последнем равенстве, получаем, что

$$
\begin{aligned}
\mathfrak{S} & =\mathfrak{S}(N)=\prod_{p} \mathscr{F}(p, N), \\
\mathscr{F}(p, N) & =1+\sum_{\alpha=1}^{\infty} \Phi\left(p^{\alpha}, N\right),
\end{aligned}
$$

Заметим, что $\mathscr{F}(p, N)$ - вещественное число. Действительно,

$$
\begin{aligned}
\overline{\Phi\left(p^{\alpha}, N\right)} & =\frac{1}{\varphi^{5}\left(p^{\alpha}\right)} \sum_{\substack{a=1 \\
(a, p)=1}}^{p^{\alpha}} \overline{T_{2}^{5}\left(a, p^{\alpha}\right)} e\left(\frac{a N}{p^{\alpha}}\right) \\
& =\frac{1}{\varphi^{5}\left(p^{\alpha}\right)} \sum_{\substack{a=1 \\
(a, p)=1}}^{p^{\alpha}}\left(\sum_{\substack{n=1 \\
(n, q)=1}}^{p^{\alpha}} e\left(-\frac{a(n+1)^{2}}{p^{\alpha}}\right)\right)^{5} e\left(\frac{a N}{p^{\alpha}}\right) \\
& =\frac{1}{\varphi^{5}\left(p^{\alpha}\right)} \sum_{\substack{a=1 \\
(a, p)=1}}^{p^{\alpha}}\left(\sum_{\substack{n=1 \\
(n, q)=1}}^{p^{\alpha}} \in\left(\frac{(-a)(n+1)^{2}}{p^{\alpha}}\right)\right)^{5} e\left(\frac{(-a) N}{p^{\alpha}}\right)=\Phi\left(p^{\alpha}, N\right) .
\end{aligned}
$$

Таким образом, особый ряд $\subseteq=\subseteq(N)$, который является бесконечным произведением по простым числам $p$ функций $\mathscr{F}(p, N)$, абсолютно сходится и является вещественным числом. В теоремах 2 и 3 , соответственно, найдены точные значения $\mathscr{F}(2, N)$ и $\mathscr{F}(p, N)$, которые определяются формулами

$$
\begin{aligned}
& \mathscr{F}(2, N)= \begin{cases}0, & \text { if } \operatorname{ord}_{2}(N) \leqslant 1, \\
\frac{26}{7}+\frac{15}{28} 2^{-\frac{3}{2}\left(\operatorname{ord}_{2}(N)-1\right)+3,}, & \text { если } \operatorname{ord}_{2}(N) \geqslant 2 \text { четно, } \\
\frac{26}{7}-\frac{13}{28} 2^{-\frac{3}{2}\left(\operatorname{ord}_{2}(N)-1\right)+3,}, & \text { есл } 2\left(N \cdot 2^{-\beta}-5\right) \neq 2, \\
\frac{26}{7}+\frac{15}{28} 2^{-\frac{3}{2}\left(\operatorname{ord}_{2}(N)-1\right)+3,}, & \operatorname{ord}_{2}\left(N \cdot 2^{-\beta}-5\right)=2, \\
\text { если } \operatorname{ord}_{2}(N) \geqslant 3 \text { нечетно, }\end{cases} \\
& \mathscr{F}_{(}(p, N)=1+\Phi(p, N)+\mathscr{F}_{1}(p, N) \text {, } \\
& \Phi(p, N)=\frac{1}{\varphi^{5}(p)}\left(-c_{p}(N-5)-10 \varepsilon_{p} p c_{p}(N-3)-5 p^{2} c_{p}(N-1)\right. \\
& \left.+5 p \delta_{p}(N-4)+10 \varepsilon_{p} \delta_{p}(N-2) p^{2}+\delta_{p}(N) p^{3}\right),
\end{aligned}
$$




$$
\mathscr{F}_{1}(p, N)=\left\{\begin{array}{cl}
0, & \text { if } \beta=0, \\
z-\frac{p}{(p-1)^{5}} & \left(1-\frac{1}{\left.p^{\frac{3}{2}(\beta-1)}\right),},\right. \\
\quad+\frac{p^{5}-p}{(p-1)^{5}\left(p^{3}-1\right)}(\text { если } \beta \geqslant 1 \text { нечетно, } \\
\frac{p^{4}}{(p-1)^{4}\left(p^{3}-1\right)}\left(1-\frac{(p+1)^{2}}{p^{\frac{3}{2}(\beta-2)+4}}\right) & \text { если } \beta \geqslant 2 \text { четно, } \\
+\frac{1+\varepsilon_{p} \delta_{p}(N)}{(p-1)^{5} p^{\frac{3}{2}(\beta-2)}}, &
\end{array}\right.
$$

где

$$
\beta=\operatorname{ord}_{p}(N)
$$

Подставляя правые части этих формул в произведение (18), можно получить точную формулу для $\mathfrak{S}(N)$ в виде произведения по простым числам, из которого следует, что ऽ $(N)=0$, при $\operatorname{ord}_{2}(N) \leqslant 1$, то есть если $N \not \equiv 0(\bmod 4)$.

В следствии 1 при $\operatorname{ord}_{2}(N) \geqslant 2$ доказано неравенство

$$
\mathscr{F}(2, N) \geqslant \frac{26}{7}-\frac{13 \sqrt{2}}{14} \geqslant \frac{13}{14}(4-\sqrt{2})>\frac{65}{28} .
$$

Соответственно, в следствиях 2, 4 и 6 найдены следующие оценки снизу:

$$
\begin{aligned}
& \mathscr{F}(p, N)>1-\frac{10}{\varphi^{2}(p)}, \quad p \geqslant 7 ; \\
& \mathscr{F}(3, N) \geqslant 1-2^{-4} ; \\
& \mathscr{F}(5, N) \geqslant 1-\frac{59}{2^{10}} .
\end{aligned}
$$

Поэтому при $\operatorname{ord}_{2}(N) \geqslant 2$

$$
\begin{aligned}
\Im(N)=\prod_{p} \mathscr{F}(p, N) & \geqslant \frac{65}{28}\left(1-2^{-4}\right)\left(1-\frac{59}{2^{10}}\right) \prod_{p \geqslant 7}\left(1-\frac{10}{\varphi^{2}(p)}\right) \\
& =\frac{65 \cdot 15 \cdot 965}{28 \cdot 16 \cdot 1024} \prod_{p \geqslant 7}\left(1-\frac{10}{\varphi^{2}(p)}\right) \\
& =\frac{940875}{458752} \prod_{p \geqslant 7}\left(1-\frac{10}{\varphi^{2}(p)}\right)>\frac{917504}{458752} \prod_{p \geqslant 7}\left(1-\frac{10}{\varphi^{2}(p)}\right) \\
& =2 \prod_{p \geqslant 7}\left(1-\frac{10}{\varphi^{2}(p)}\right) .
\end{aligned}
$$

\section{Список литературы}

1. Hua L. K., Some results in the additive prime number theory. Quarterly J. Math. (1938) 9, 68-80.

2. Коробов Н. М., Тригонометрические суммы и их приложения. Наука, Москва, 1989. 